\title{
Ultimate storage ring based on fourth-order geometric achromats
}

\author{
Yunhai Cai, Karl Bane, Robert Hettel, Yuri Nosochkov, and Min-Huey Wang \\ SLAC National Accelerator Laboratory, Menlo Park, California 94025, USA \\ Michael Borland \\ Argonne National Laboratory, Argonne, Illinois 60439, USA \\ (Received 2 December 2011; published 23 May 2012)
}

\begin{abstract}
We have designed an "ultimate" storage ring for a future light source that would be resided in the positron-electron-project (PEP) tunnel and achieves the diffraction-limited emittances (at $1.5 \AA$ ) of $12 \mathrm{pm}-\mathrm{rad}$ in both horizontal and vertical planes with a 4.5-GeV electron beam. These emittances include the contribution of intrabeam scattering at a nominal current of $200 \mathrm{~mA}$ in 3300 bunches. This quality beam in conjunction with a conventional 4-m undulator in a straight section can generate synchrotron radiation having a spectral brightness above $10^{22}\left[\right.$ photons $\left./ \mathrm{s} / \mathrm{mm}^{2} / \mathrm{mrad}^{2} / 0.1 \% \mathrm{BW}\right]$ at a $10 \mathrm{keV}$ photon energy. The high coherence at the diffraction limit makes this design competitive with 4th generation light sources based on an energy recovery linac. In addition, the beam lifetime is several hours and the dynamic aperture is large enough to allow off-axis injection. The alignment and stability tolerances, though challenging, are achievable. A ring with all these properties is only possible because of several major advances in mitigating the effects of nonlinear resonances.
\end{abstract}

PACS numbers: 41.75.Fr, 29.27. $-\mathrm{a}, 41.85 .-\mathrm{p}, 42.72 .-\mathrm{g}$

\section{INTRODUCTION}

An ultimate storage ring (USR) [1], defined as an electron ring-based light source having an emittance in both transverse planes at the diffraction limit for the range of $x$-ray wavelengths of interest for a scientific community, would provide very high brightness photons having high transverse coherence that would extend the capabilities of $\mathrm{x}$-ray imaging and probe techniques beyond today's performance. It would be a cost-effective, high-coherence 4th generation light source [2], competitive with one based on energy recovery linac (ERL) technology [3], serving a large number of users studying material, chemical, and biological sciences. Furthermore, because of the experience accumulated over many decades of ring operation, it would have the great advantage of stability and reliability. Given that there are three predominant spectral ranges of interest for the photon science community roughly specified as $\geq 6 \AA$ (with electron energy $\approx 2 \mathrm{GeV}$ ), $6-0.5 \AA$ (with electron energy 3 to $5 \mathrm{GeV}$ ), and $\leq 0.5 \AA$ (with electron energy $\geq 6 \mathrm{GeV}$ ), USRs having diffraction-limited emittances (defined as $\lambda / 4 \pi$ for wavelength $\lambda$ ) on the scale of 100,10 , and 1 pm-rad can be considered.

In this paper we consider the design of a midenergy USR having 10-pm-rad emittance. It is a tremendous challenge to design a storage ring having such an extremely low

Published by the American Physical Society under the terms of the Creative Commons Attribution 3.0 License. Further distribution of this work must maintain attribution to the author(s) and the published article's title, journal citation, and DOI. emittance, a factor of 100 smaller than those in existing midenergy light sources, especially such that it has adequate dynamic aperture and beam lifetime. In many ultralow emittance designs [4-8], the injection acceptances are not large enough for accumulation of the electron beam, necessitating on-axis injection where stored electron bunches are completely replaced with newly injected ones. Recently, starting with the MAX-IV 7-bend achromatic cell [9], we have made significant progress $[10,11]$ with the design of positron-electron-project (PEP)-X, a USR that would inhabit the decommissioned PEP-II tunnel at SLAC (Fig. 1). The enlargement of the dynamic aperture is largely a result of the cancellations [12] of the 4th-order resonances in the 3rd-order achromats [13] and the effective use of lattice optimization programs $[14,15]$.

In this paper, we will show those cancellations of the 4th-order resonances using an analytical approach based on the exponential Lie operators and the Poisson brackets. Wherever possible, our analytical results will be compared with their numerical counterparts [12]. Using the derived formulas, we will construct 4th-order geometric achromats and use them as modules for the lattice of the PEP-X USR, noting that only geometric terms are canceled to the 4th order

We begin with a review of spontaneous synchrotron radiation emitted from an undulator in an electron storage ring in Sec. II and evaluate the performances of PEP-X as a diffraction-limited light source based on the lattice and beam parameters. We will outline our lattice design in Sec. III where both linear optics and nonlinear analysis will be considered and presented in an integrated and coherent fashion. In Sec. IV, we will present the performance of the 


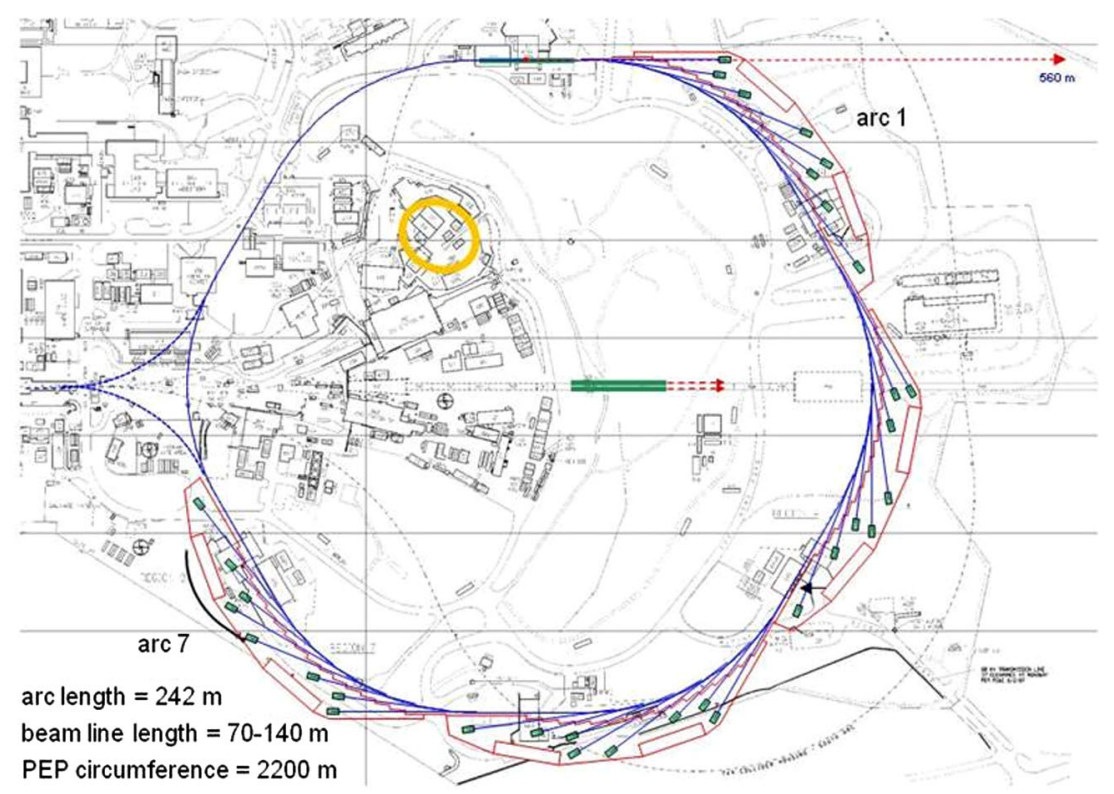

FIG. 1. Layout of PEP-X at SLAC as an ultimate storage ring.

design lattice along with the analysis of the machine tolerances and single-particle dynamics.

Continuing into Sec. V, we will consider the scattering effects within the electron bunch. Finally, we will briefly estimate several important collective instabilities in Sec. VI and will discuss aspects of our design in the conclusion.

\section{SPONTANEOUS SYNCHROTRON RADIATION}

The wavelength of synchrotron radiation at the $n$th harmonic from an electron beam in a planar undulator having a period of $\lambda_{u}$ and a peak magnetic field $B_{0}$ is given by

$$
\lambda_{n}=\frac{\lambda_{u}}{2 n \gamma^{2}}\left(1+K^{2} / 2\right), \quad(n=1,3,5, \ldots),
$$

where $\gamma$ is the Lorentz relativistic factor and $K=$ $e B_{0} \lambda_{u} / 2 \pi m c$ is the undulator strength parameter. The bandwidth of the spectral line at the $n$th harmonic is inversely proportional to the product of the number of undulator periods $N_{u}$ and the harmonic number $n$,

$$
\frac{\Delta \omega}{\omega_{n}} \approx \frac{1}{n N_{u}}
$$

The angle-integrated photon spectral flux in the forward direction is proportional to the electron beam current $I$ and can be written as [16]

$$
\mathcal{F}_{n}=\frac{\pi}{2} \alpha N_{u} \frac{\Delta \omega}{\omega} \frac{I}{e} Q_{n}\left(\frac{n K^{2}}{4+2 K^{2}}\right) .
$$

Here $\alpha$ is the fine structure constant and the function $Q_{n}(Y)$ is defined by

$$
Q_{n}(Y)=4 Y\left[J_{(n+1) / 2}(Y)-J_{(n-1) / 2}(Y)\right]^{2},
$$

where $J_{m}$ are the Bessel functions. To achieve a higher flux, one of the important performance parameters for a light source, one needs a higher current, a longer undulator, and a reasonable value of the undulator strength $K$, as illustrated in Fig. 2. As an example, we calculate the photon spectral flux using the PEP-X parameters in Table I and show the results in Fig. 3.

Another important aspect of a light source is its spectral brightness $\mathcal{B}_{n}$, defined as the ratio of the photon spectral flux to the volume of the convoluted phase of the electron beam and the photon beam in the two transverse dimensions, namely, [16],

$$
\mathcal{B}_{n}=\frac{\mathcal{F}_{n}}{4 \pi^{2} \Sigma_{x} \Sigma_{x}^{\prime} \Sigma_{y} \Sigma_{y}^{\prime}}
$$

where the convoluted sizes and divergences are

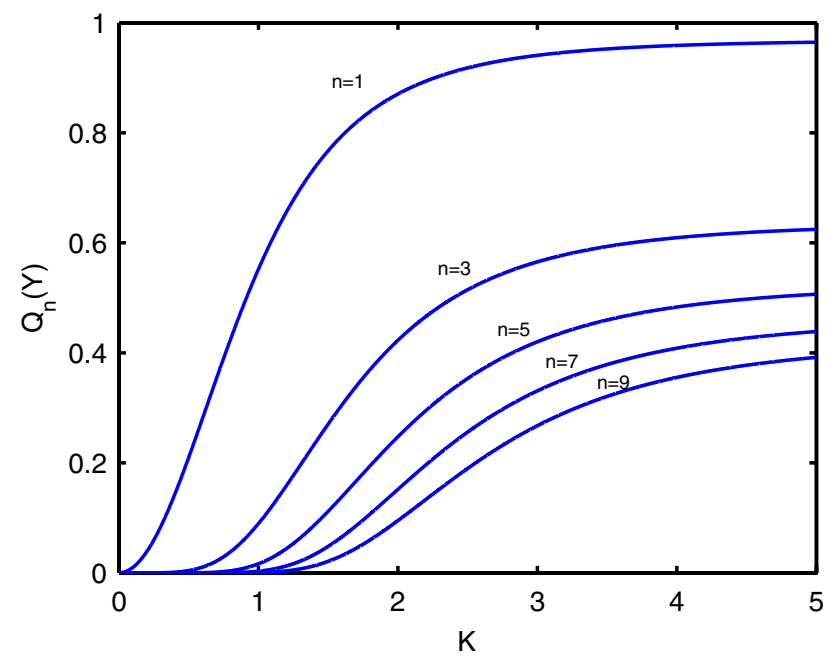

FIG. 2. Function $Q_{n}\left(\frac{n K^{2}}{4+2 K^{2}}\right)$. 
TABLE I. Main parameters of PEP-X as an ultimate storage ring. The effects of the intrabeam scattering and $90 \mathrm{~m}$ of damping wigglers are included.

\begin{tabular}{lcc}
\hline \hline Parameter & Description & Value \\
\hline$E[\mathrm{GeV}]$ & Beam energy & 4.5 \\
$I[\mathrm{~mA}]$ & Beam current & 200 \\
$\epsilon_{x, y}[\mathrm{pm}-\mathrm{rad}]$ & $x, y$ Emittances & $11.5,11.5$ \\
$\sigma_{\delta}$ & Energy spread & $1.25 \times 10^{-3}$ \\
$\beta_{x, y}[\mathrm{~m}]$ & $x, y$ Beta functions at ID & $4.92,0.8$ \\
$\lambda_{u}[\mathrm{~cm}]$ & Period of undulator & 2.3 \\
$L_{u}[\mathrm{~m}]$ & Length of undulator & 4.4 \\
$K$ & Undulator strength & 2.26 \\
\hline \hline
\end{tabular}

$$
\begin{gathered}
\Sigma_{x, y}=\sqrt{\sigma_{x, y}^{2}+\sigma_{\lambda}^{2}}, \\
\Sigma_{x, y}^{\prime}=\sqrt{\sigma_{x, y}^{\prime 2}+\sigma_{\lambda}^{\prime 2}} .
\end{gathered}
$$

Here $\sigma_{x, y}, \sigma_{x, y}^{\prime}$ are the rms sizes and divergences of the electron beam, respectively. Given the undulator length $L_{u}=N_{u} \lambda_{u}$, the size and divergence of the photon beam are given by

$$
\begin{gathered}
\sigma_{\lambda}=\sqrt{\frac{\lambda_{n} L_{u}}{8 \pi^{2}}} \\
\sigma_{\lambda}^{\prime}=\sqrt{\frac{\lambda_{n}}{2 L_{u}}} .
\end{gathered}
$$

It is worth noting that the "emittance" of the photon beam,

$$
\epsilon_{\lambda}=\sigma_{\lambda} \sigma_{\lambda}^{\prime}=\lambda_{n} / 4 \pi
$$

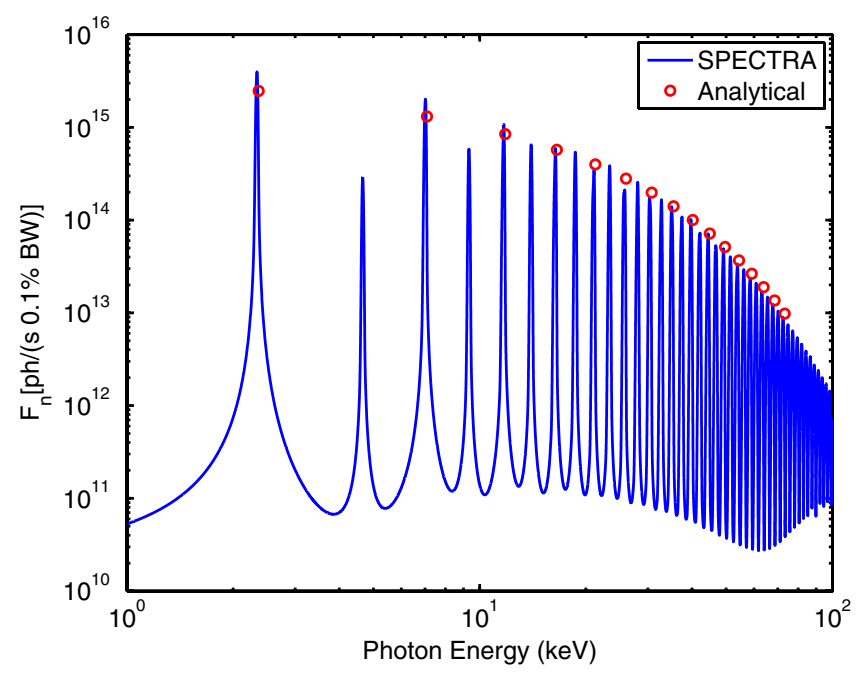

FIG. 3. Photon spectral flux in a $0.1 \%$ bandwidth calculated using SPECTRA [48] within the forward cone of $3 \sigma_{\lambda_{1}}^{\prime}$ and the formula [Eq. (2.3)]. depends only on its wavelength. Accordingly, its "beta" function is given by

$$
\beta_{\lambda}=\frac{\sigma_{\lambda}}{\sigma_{\lambda}^{\prime}}=\frac{L_{u}}{2 \pi}
$$

One can easily show that the convoluted phase space area, $2 \pi \Sigma_{x, y} \Sigma_{x, y}^{\prime}$, is at a minimum if the beta functions of the electron beam are matched to those of the photon beam, namely, $\beta_{x, y}=\beta_{\lambda}$. The matching conditions not only maximize the brightness but also simplify its formula to be

$$
\mathcal{B}_{n}^{(m)}=\frac{\mathcal{F}_{n}}{4 \pi^{2}\left(\epsilon_{x}+\lambda_{n} / 4 \pi\right)\left(\epsilon_{y}+\lambda_{n} / 4 \pi\right)},
$$

where $\epsilon_{x, y}$ are the emittances of the electron beam in the horizontal and vertical planes, respectively. Reducing electron emittance increases brightness towards an ultimate upper limit for spontaneous radiation from an undulator:

$$
\mathcal{B}_{n}^{(u)}=\frac{4 \mathcal{F}_{n}}{\lambda_{n}^{2}} .
$$

While beam brightness can be increased by reducing electron emittance in the denominator of Eq. (2.12), this emittance reduction may also lead to a reduction in the achievable flux in the numerator due to collective instabilities in the electron beam. The maximum brightness for angstrom-level spontaneous radiation, the wavelengths of interest for studying the molecular structure and properties of materials, from a particular insertion device in a storage ring is thus due to the trade-off between low emittance and achievable beam current.

So far, we have ignored the emittance of the electron beam in the longitudinal dimension. Actually, the energy spread of the beam adds to the width of the undulator's spectral lines, thereby reducing spectral brightness. Assuming the electron beam has a Gaussian distribution in the relative energy $\delta(=d E / E)$ given by

$$
\rho(\delta)=\frac{1}{\sqrt{2 \pi} \sigma_{\delta}} e^{-\left(\delta^{2} / 2 \sigma_{\delta}^{2}\right)},
$$

then the frequency dependence at the $n$th harmonic is also a narrow Gaussian with a sigma of $\sigma_{\omega_{n}}=\omega_{n} / \sqrt{2} n N_{u}$, which is consistent with Eq. (2.2). The reduction factor $f_{\delta}$ can then be estimated by

$$
f_{\delta}=\frac{1}{\sqrt{2 \pi} \sigma_{\delta}} \int_{-\infty}^{\infty} e^{-\left(\Delta \omega_{n}^{2} / 2 \sigma_{\omega_{n}}^{2}\right)} e^{-\left(\delta^{2} / 2 \sigma_{\delta}^{2}\right)} d \delta,
$$

where $\Delta \omega_{n}=2 \omega_{n} \delta$, which can be derived from Eq. (2.1). Carrying out the integral, we obtain

$$
f_{\delta}=\frac{1}{\sqrt{1+8\left(\sigma_{\delta} n N_{u}\right)^{2}}} .
$$

For a large harmonic number $n$ or a large number of undulator periods $N_{u}$, the reduction becomes significant. In a typical electron storage ring having relative energy 


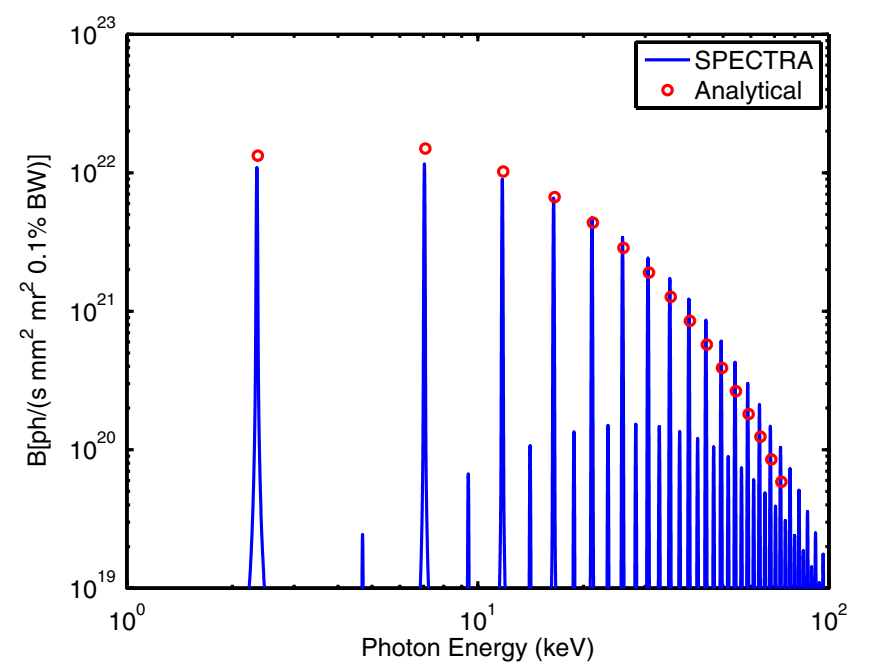

FIG. 4. Spectral brightness of PEP-X at $200 \mathrm{~mA}$, calculated using SPECTRA and the formula [Eq. (2.5)] multiplying by the reduction factor [Eq. (2.16)].

spread $\sigma_{\delta}=0.001$, the degradation of brightness at higher harmonics constrains the useful number $N_{u}$ of bandwidthreducing undulator periods to no more than a couple of hundred. Moreover, the energy spread indirectly limits the photon flux since it is proportional to $N_{u}$.

To give an example of a synchrotron light source reaching the diffraction limit at $1-\AA$ wavelength, we use the PEP-X parameters tabulated in Table I and calculate the spectral brightness as shown in Fig. 4. In the figure, one can see a comparison of the results generated by SPECTRA and the analytical formulas outlined in this section. At the peaks of the odd harmonics, the agreement between the numerical code and the analytical approach is excellent.

Finally, as the emittances approach the diffraction limit, namely $\epsilon_{x, y} \approx \lambda_{n} / 4 \pi$, the synchrotron light has more coherence in the transverse dimensions. One can define the fraction of coherence,

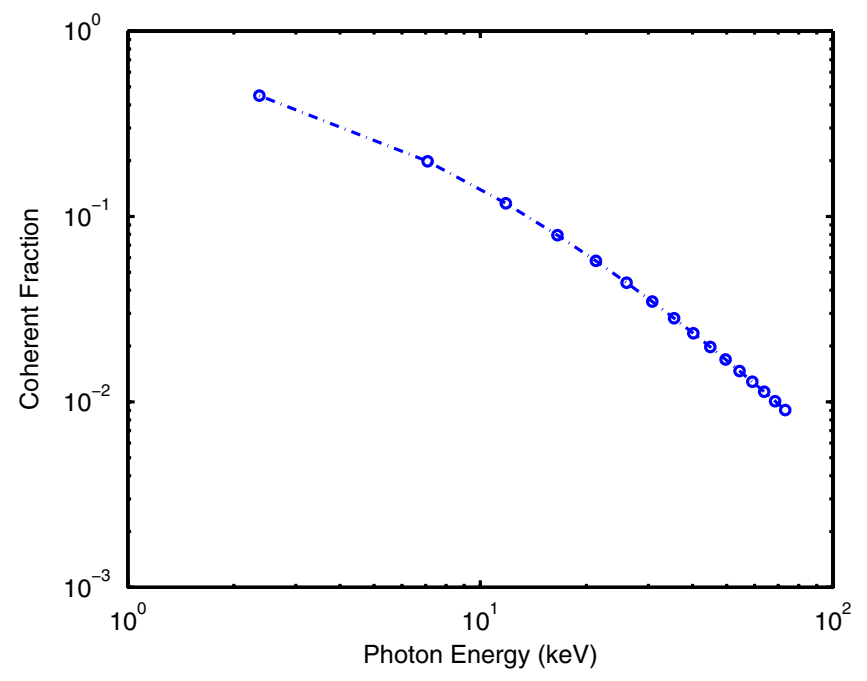

FIG. 5. Coherent fraction for the PEP-X ultimate storage ring.

$$
f_{\mathrm{coh}}=\frac{\mathcal{B}_{n}}{\mathcal{B}_{n}^{(u)}}=\frac{\lambda_{n}^{2}}{16 \pi^{2} \Sigma_{x} \Sigma_{x}^{\prime} \Sigma_{y} \Sigma_{y}^{\prime}},
$$

to quantify the degree of coherence. Using the parameters in Table I, we calculate the coherent fraction and plot it in Fig. 5. One can see that the PEP-X design provides extremely high-coherence multi-keV $\mathrm{x}$ rays.

It is clear from the figures of photon flux, spectral brightness, and coherent fraction that PEP-X would be a future light source superior to existing facilities such as PETRA-III [17] or projects under construction [9,18]. It would also be competitive with the other future light sources [2] based on an ERL.

\section{LATTICE DESIGN}

To reach the diffraction limit of an angstrom, the lattice of the PEP-X ultimate storage ring must yield a very low electron emittance on the order of $10 \mathrm{pm}$-rad at $4.5 \mathrm{GeV}$ beam energy, while providing dispersion-free optics for insertion devices (IDs) and sufficient dynamic aperture for injection and beam lifetime. The cancellation of dispersion minimizes the electron beam size within the ID, thus leading to enhanced brightness of the photon beam. An additional constraint is that PEP-X must fit into the existing 2.2-km PEP-II tunnel and therefore must adopt the PEP-II ring layout with six $243 \mathrm{~m}$ long arcs and six $123 \mathrm{~m}$ long straight sections as shown in Fig. 1. The PEP-X arcs have identical lattices comprised of periodic cells, and the long straights are made of nearly periodic alternating focusing (F) and defocusing (D) quadupoles separated equally in space (FODO) cells, except in the injection

TABLE II. PEP-X lattice parameters with damping wigglers at zero beam current.

\begin{tabular}{lc}
\hline \hline Parameter & Value \\
\hline Energy, $E[\mathrm{GeV}]$ & 4.5 \\
Circumference, $\mathcal{C}[\mathrm{m}]$ & 2199.32 \\
Tune, $\nu_{x}, \nu_{y}, \nu_{s}$ & $113.23,65.14,0.0069$ \\
Emittance, $\epsilon_{0 w}[\mathrm{pm} \cdot \mathrm{rad}]$ & 11.0 \\
Bunch length, $\sigma_{z}[\mathrm{~mm}]$ & 3.0 \\
Energy spread, $\sigma_{\delta}$ & $1.20 \times 10^{-3}$ \\
Momentum compaction & $4.96 \times 10^{-5}$ \\
Damping partition number, $I_{x}, I_{y}, I_{s}$ & $1.175,1.00,1.825$ \\
Damping time, $\tau_{x}, \tau_{y}, \tau_{s}[\mathrm{~ms}]$ & $19,22,12$ \\
Natural chromaticity, $\xi_{x 0}, \xi_{y 0}$ & $-162.3,-130.1$ \\
Energy loss per turn, $U_{0}[\mathrm{MeV}]$ & 2.95 \\
rf voltage, $V_{\mathrm{rf}}[\mathrm{MV}]$ & 8.3 \\
rf frequency, $f_{\mathrm{rf}}[\mathrm{MHz}]$ & 476 \\
Harmonic number & 3492 \\
Wiggler length, $L_{w}[\mathrm{~m}]$ & 89.66 \\
Wiggler period, $\lambda_{w}[\mathrm{~cm}]$ & 5.0 \\
Wiggler peak field, $B_{w}[\mathrm{~T}]$ & 1.5 \\
Length of ID straight, $L_{\mathrm{ID}}[\mathrm{m}]$ & 5.0 \\
Beta at ID center, $\beta_{x}, \beta_{y}[\mathrm{~m}]$ & $4.92,0.80$ \\
\hline \hline
\end{tabular}


straight. The latter has special optics with large $\beta_{x}=$ $200 \mathrm{~m}$ at the injection septum for maximum acceptance of the injected beam. Although, in general, the injection optics breaks the 6-fold ring periodicity, the effective periodicity was restored by making the injection straight linear matrix the same as in the other straights. This required that the fractional part of the phase advance in all straights is the same. This helps to suppress some of the ring systematic resonances and increase the dynamic aperture to the level of an ideal 6-fold ring aperture. The complete list of PEP-X lattice parameters with the wiggler is shown in Table II. Lattice of the FODO and injection straight sections have not significantly changed since the PEP-X baseline design, and its description can be found in the report [19].

\section{A. Arc optics}

As a result of the balance between quantum excitation and radiation damping, an electron beam in storage rings reaches an equilibrium distribution with horizontal emittance given by [20]

$$
\epsilon_{x}=C_{q} \frac{\gamma^{2}}{I_{x}} \frac{I_{5}}{I_{2}}
$$

with

$$
C_{q}=\frac{55}{32 \sqrt{3}} \frac{\hbar}{m c}, \quad I_{2}=\oint \frac{d s}{\rho^{2}}, \quad I_{5}=\oint \frac{\mathcal{H}_{x}}{\rho^{3}} d s,
$$

where

$$
\mathcal{H}_{x}=\beta_{x} \eta_{x}^{\prime 2}+2 \alpha_{x} \eta_{x} \eta_{x}^{\prime}+\gamma_{x} \eta_{x}^{2},
$$

$I_{x}$ is the horizontal damping partition number, $\rho$ the bending radius, $\eta_{x}, \eta_{x}^{\prime}$ are the horizontal dispersion and its slope, and $\beta_{x}, \alpha_{x}, \gamma_{x}$ the horizontal Courant-Snyder parameters.

For a simple ring with identical arc cells and without damping wiggler, the above dependence can be simplified to

$$
\epsilon_{0}=C_{q} \gamma^{2} \theta^{3} \frac{F}{I_{x}}
$$

where the parameter $F=I_{5} /\left(I_{2} \theta^{3}\right)$ depends on lattice functions in the cell dipoles, and $\theta$ is a bending angle per dipole. For a minimal emittance, it is therefore desired to have a large number of short cells with small $\theta$ and cell lattice with low $F$ value. Note that due to the strong dependence on $\theta$, the rings with longer arcs have a significant advantage. For comparison, the total length of PEP-X arcs is about $2 / 3$ of the ring circumference, namely $1460 \mathrm{~m}$.

Optics of the theoretical minimum emittance [21] (TME) cell can yield the lowest possible emittance corresponding to the minimum value of $F$ :

$$
F_{\min }^{(\mathrm{TME})}=\frac{1}{12 \sqrt{15}} .
$$

This, however, requires a very strong focusing which may limit dynamic aperture, hence in practical designs the TME lattice is usually set to a higher $F$ value. Unfortunately, the TME cells are not suitable for insertion devices due to lack of dispersion-free straights. On the other hand, double bend achromat [22] (DBA) cells, widely used in light source rings, provide the ID dispersion-free straights, but their minimal natural emittance is a factor of 3 higher relative to a TME cell with the same bending angle. A compromise solution to obtain both the low emittance and dispersion-free straight is a hybrid cell- the so-called multibend achromat (MBA) comprised of several short TME-type units at the cell center and a dispersion matching unit (similar to half DBA) at each cell end.

The compact cell design requires both optical and engineering solutions. An example is the MAX-IV 7-bend achromat cell [9], where the standard TME defocusing quadrupoles are eliminated and replaced by a defocusing gradient in the dipoles, and the sextupole magnets are integrated with dipoles or quadrupoles in compact blocks. A defocusing gradient in the dipole has the added advantage of higher $I_{x}$ for even lower emittance.

The PEP-X MBA cell with 7 dipoles, shown in Fig. 6, is similar to the MAX-IV cell with a few modifications. It has a natural emittance of $\epsilon_{0}=29.0$ pm-rad at $4.5 \mathrm{GeV}$ and zero current. The factor $F / I_{x}$ in this lattice is about 5.7 times higher than in an equivalent minimum emittance TME cell due to the choice of a relatively low phase advance required for large dynamic aperture. The chosen cell phase advance is $\mu_{x}=4 \pi+\pi / 4$ and $\mu_{y}=$ $2 \pi+\pi / 4$, and the cell length is matched to $30.4 \mathrm{~m}$. This provides an optimal linear cell optics and yields 8 cells per arc resulting in an identity linear transformation per each arc. The reason for such a choice will be given in the next section. The TME units have periodic lattice functions and are made of a focusing quadrupole and a dipole with defocusing gradient per unit. A matching dipole at each cell end is gradient free and $20 \%$ shorter than the TME dipole. The ID straight length is $L_{\mathrm{ID}}=5 \mathrm{~m}$, and $\beta_{x} / \beta_{y}=$ $4.9 / 0.8 \mathrm{~m}$ at the ID center. The $\beta_{y}$ at ID is near its optimal value of $L_{u} / 2 \pi$ for maximum brightness. Compared to MAX-IV, this cell has 4 additional matching quadrupoles for a larger tuning range of the ID $\beta$ functions. In particular, the ID $\beta_{y}$ can be varied up to a $5 \mathrm{~m}$ value while the cell phase advance is fixed and the ID $\beta_{x}$ and cell emittance are not significantly changed.

An ultralow emittance lattice such as that of PEP-X is characterized by very small beta functions and dispersion, achieved with many quadrupoles and resulting in a very large natural chromaticity. To correct the chromaticity, the chromatic sextupole magnets become extremely strong as the dispersion gets smaller and smaller. The nonlinear effects generated by such strong sextupoles result in a severe reduction of dynamic aperture. An efficient minimization of these nonlinearities becomes essential for a successful design of an ultimate storage ring. 


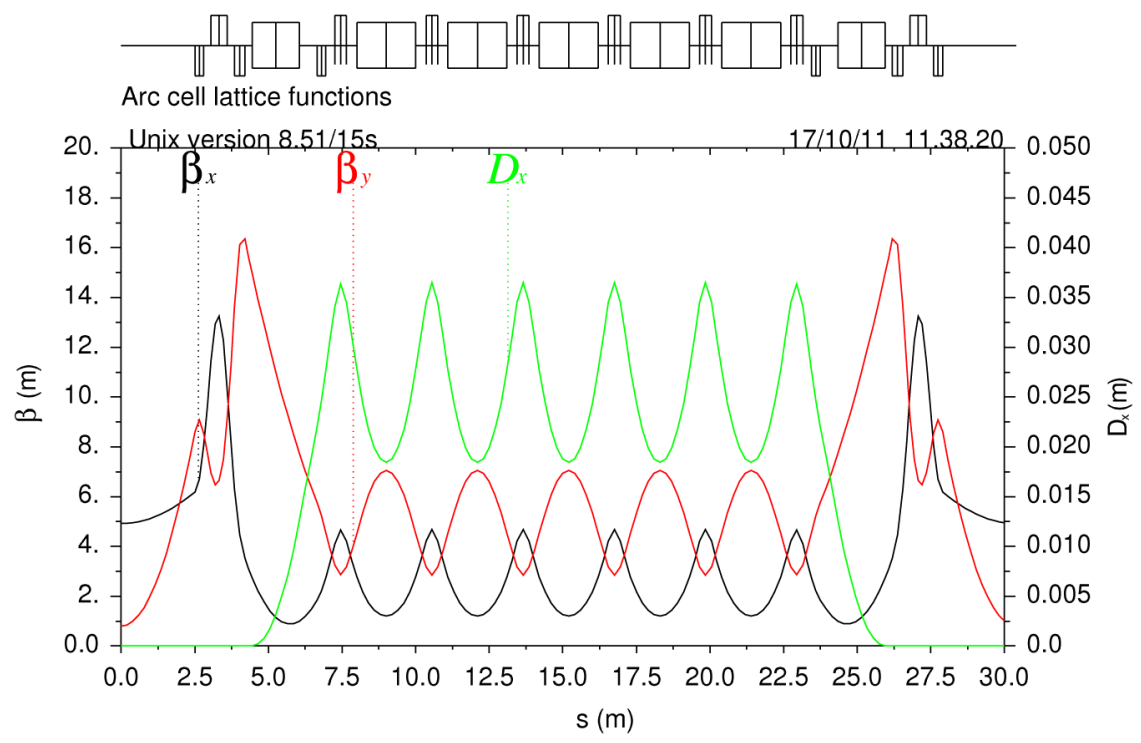

FIG. 6. Lattice functions in PEP-X MBA cell with 7 dipoles. Four quadrupoles on each side of the ID straight can be used for variation of ID beta functions.

The cell sextupole scheme consists of 4 families of chromatic sextupoles and 6 harmonic sextupoles. The chromaticity-correcting sextupoles are placed at the center of the TME focusing quadrupoles and at each end of the dipole where dispersion is not zero. The harmonic sextupoles are placed within the two dispersion-free quadrupole triplets adjacent to the ID straight. This scheme provides sufficient flexibility for optimization of nonlinear chromaticity and amplitude dependence of the betatron tunes from the sextupole perturbations.

The cell magnet parameters are within a reasonable range. At $4.5 \mathrm{GeV}$, the dipole field is $<1.5 \mathrm{kG}$, the quadrupole field is $<8.6 \mathrm{kG}$ at $20 \mathrm{~mm}$ radius, and the sextupole field is $<7.8 \mathrm{kG}$ at $15 \mathrm{~mm}$ radius and $0.2 \mathrm{~m}$ sextupole length.

\section{B. Perturbation of sextupoles}

We would like to provide a general treatment of sextupole perturbation and then apply it to the design of PEP-X. For simplicity, we start with the geometric aberrations. Let us consider a set of thin-lens sextupoles at position $i=1, \ldots, n$ in a beam line. Between any two adjacent sextupoles at positions $i-1$ and $i$, we have a linear transfer map $\mathcal{M}_{i-1, i}$. Based on the Lie algebra method [23], the transfer map $\mathcal{M}$ of the beam line can be written as [24]

$$
\mathcal{M}=\mathcal{M}_{0,1} e^{-: \mathcal{V}_{1}(\vec{z}):} \mathcal{M}_{1,2} e^{-: \mathcal{V}_{2}(\vec{z}):} \ldots \mathcal{M}_{n-1, n} e^{-: \mathcal{V}_{n}(\vec{z}):} \mathcal{M}_{n, n+1}
$$

where $\vec{z}$ is a vector in the four-dimensional transverse phase space and the vector potential $\mathcal{V}_{i}(\vec{z})=S_{i}\left(x^{3}-3 x y^{2}\right) / 6$. Here we have denoted $S_{i}$ as an integrated strength,

$$
S_{i}=\frac{L_{i}}{(B \rho)} \frac{\partial^{2} B_{y}}{\partial x^{2}},
$$

where $L_{i}$ is the length of the sextupole magnet and $(B \rho)$ the magnetic rigidity. By repeatedly applying a similarity transformation,

$$
\mathcal{L}^{-1} e^{-: \mathcal{F}(\vec{z})}: \mathcal{L}=e^{-: \mathcal{F}\left(\mathcal{L}^{-1} \vec{z}\right)},
$$

where $\mathcal{L}$ is a linear map and $\mathcal{F}$ is an arbitrary function, one can show [24]
$\mathcal{M}=\mathcal{M}_{0, n+1} e^{-: \mathcal{V}_{1}\left(\mathcal{M}_{1, n+1}^{-1} \bar{z}\right)}: e^{-: \mathcal{V}_{2}\left(\mathcal{M}_{2, n+1}^{-1} \bar{z}\right)} \ldots e^{-: \mathcal{V}_{n}\left(\mathcal{M}_{n, n+1}^{-1} \bar{z}\right):}$

where $\mathcal{M}_{i, n+1}=\mathcal{M}_{i, i+1} \ldots \mathcal{M}_{n, n+1}$ is the linear transfer map from position $i$ to $n+1$ and the superscript " -1 " is used to denote its inverse map. One can see from Eq. (3.9) that the total map $\mathcal{M}$ is factorized into the linear map $\mathcal{M}_{0, n+1}$ and a nonlinear map,

$$
e^{-: \mathcal{V}_{1}\left(\mathcal{M}_{1, n+1}^{-1} \vec{z}\right):} e^{-: \mathcal{V}_{2}\left(\mathcal{M}_{2, n+1}^{-1} \vec{z}\right)} \ldots e^{-: \mathcal{V}_{n}\left(\mathcal{M}_{n, n+1}^{-1} \vec{z}\right):}
$$

and more importantly all nonlinearities are effectively transported to the end of the beam line. 
It is well known that one can use a linear symplectic map $\mathcal{A}^{-1}$ to make a coordinate transformation to the normalized coordinates. As a consequence, the linear transfer map can be decomposed into $\mathcal{M}_{i, n+1}=\mathcal{A}_{i}^{-1} \mathcal{R}_{i, n+1} \mathcal{A}_{n+1}$, where
$\mathcal{R}_{i, n+1}$ is a rotational map with the betatron phase advances $\mu_{x, i}, \mu_{y, i}$ as the angles. Using the normalized coordinates and repeatedly applying the similarity transformation [Eq. (3.8)], we can rewrite the map of the beam line as

$$
\mathcal{M}=\mathcal{A}_{0}^{-1} \mathcal{R}_{0, n+1} e^{-: \mathcal{V}_{1}\left(\mathcal{R}_{1, n+1}^{-1} \mathcal{A}_{1} \vec{z}\right):} e^{-: \mathcal{V}_{2}\left(\mathcal{R}_{2, n+1}^{-1} \mathcal{A}_{2} \vec{z}\right)} \ldots e^{-: \mathcal{V}_{n}\left(\mathcal{R}_{n, n+1}^{-1} \mathcal{A}_{n} \vec{z}\right):} \mathcal{A}_{n+1}
$$

Explicitly, we have

$$
\mathcal{V}_{i}\left(\mathcal{R}_{i, n+1}^{-1} \mathcal{A}_{i} \vec{z}\right)=\frac{S_{i} \sqrt{\beta_{x, i}}}{6}\left(\beta_{x, i} x_{i}^{3}-3 \beta_{y, i} x_{i} y_{i}^{2}\right),
$$

where $\beta_{x, i}, \beta_{y, i}$ are the optical beta functions at position $i$, $x_{i}=x \cos \mu_{x, i}-p_{x} \sin \mu_{x, i}$, and $y_{i}=y \cos \mu_{y, i}-p_{y} \sin \mu_{y, i}$. In fact, $x_{i}, y_{i}$ are the normalized coordinates respectively in the horizontal and vertical planes at position $i$.

So far, we have not yet made any approximations. To carry out the perturbation theory of the sextupoles, we need to combine the Lie factors $e^{-\mathcal{V}_{1}} \ldots e^{-\mathcal{V}_{n}}$ in Eq. (3.11) into a single Lie operator. This can be achieved by repeatedly applying the Cambell-Baker-Hausdorf theorem $e^{: \mathcal{A}:} e^{: \mathcal{B}:}=e^{: \mathcal{C}:}$, where $\mathcal{C}=\mathcal{A}+\mathcal{B}+\{\mathcal{A}, \mathcal{B}\}_{\mathrm{PB}} / 2+\cdots$. Here the bracket with the subscript "PB" denotes the well-known Poisson bracket. For the perturbation of the sextupole strength $S$, we obtain

$$
\mathcal{M}=\mathcal{A}_{0}^{-1} \mathcal{R}_{0, n+1} e^{: f_{3}+f_{4}+\ldots:} \mathcal{A}_{n+1}
$$

where

$$
f_{3}=-\sum_{i=1}^{n} \mathcal{V}_{i}
$$

and

$$
f_{4}=\frac{1}{2} \sum_{i=1}^{n} \sum_{j>i}^{n}\left\{\mathcal{V}_{i}, \mathcal{V}_{j}\right\}_{\mathrm{PB}} .
$$

Clearly, $f_{3}$ is of the first order of $S$ and $f_{4}$ of the second order. Similar to the Hamiltonian perturbation theory [25], $f_{3}$ gives the driving term of the third-order resonances provided that the action-angle variables, $x=\sqrt{2 J_{x}} \cos \phi_{x}$, $p_{x}=-\sqrt{2 J_{x}} \sin \phi_{x}, \quad y=\sqrt{2 J_{y}} \cos \phi_{y}, \quad$ and $\quad p_{y}=$ $-\sqrt{2 J_{y}} \sin \phi_{y}$ are used.

The Poisson bracket of any pair of $\mathcal{V}_{i, j}$ can be computed easily and the result is given by

$\left\{\mathcal{V}_{i}, \mathcal{V}_{j}\right\}_{\mathrm{PB}}=S_{i} S_{j} \sqrt{\beta_{x, i} \beta_{x, j}}\left[\sin \left(\mu_{y, i}-\mu_{y, j}\right) \beta_{y, i} \beta_{y, j} x_{i} x_{j} y_{i} y_{j}+\sin \left(\mu_{x, i}-\mu_{x, j}\right)\left(\beta_{x, i} x_{i}^{2}-\beta_{y, i} y_{i}^{2}\right)\left(\beta_{x, j} x_{j}^{2}-\beta_{y, j} y_{j}^{2}\right) / 4\right]$

Clearly, all terms in the brackets are octupole like, namely a fourth-order monomial in $x, p_{x}, y$, and $p_{y}$. It is worth noting that this bracket vanishes when the phase differences in both planes are integers of $\pi$.

Essentially, we have worked out the first and, more importantly, the second-order sextupole perturbation using the Lie method. Our assumption of a thin lens could be removed since one can always divide a thick sextupole into many thin slices and then apply the thin-lens formulas. In practice, it is sometimes easier to compute [12] $f_{3}$ and $f_{4}$ numerically using the differential algebra [26] and DragtFinn factorization [27].

\section{One family of sextupoles in arc}

Now we can apply the general results of the perturbation theory to the arcs of PEP-X. As we mentioned in a previous section, we designed a periodical cell with betatron phase advances, $\mu_{x}=4 \pi+\pi / 4$ and $\mu_{y}=2 \pi+\pi / 4$, in the horizontal and the vertical planes, respectively. For the linear optics, every eight of such cells makes an identity transformation and form an achromat. In the design lattice, every arc consists of one such achromat. We would like to explain why this choice was made.

Let us study an achromat that consists of eight PEP-X cells and each cell has a thin sextupole at the same location. It is well known that $f_{3}=0$ in the achromat [13]. This can also be shown directly using Eqs. (3.12) and (3.14). As a result, this beam line preserves the property of an achromat, even at a nonlinear level, up to the first order of sextupole strength.

To proceed to the next order, we need to compute $f_{4}$ using Eqs. (3.15) and (3.16) and add up the contributions from all 28 brackets. Expressing the result in terms of the complex coordinates, $x=\left(a_{x}+i a_{x}^{+}\right) / \sqrt{2}, \quad p_{x}=$ $\left(i a_{x}+a_{x}^{+}\right) / \sqrt{2}, y=\left(a_{y}+i a_{y}^{+}\right) / \sqrt{2}, p_{y}=\left(i a_{y}+a_{y}^{+}\right) / \sqrt{2}$, we have

$$
\begin{aligned}
f_{4}= & -\frac{1}{4} S^{2} \beta_{x}\left\{(1+2 \sqrt{2})\left[\beta_{x}^{2}\left(a_{x} a_{x}^{+}\right)^{2}+\beta_{y}^{2}\left(a_{y} a_{y}^{+}\right)^{2}\right]\right. \\
& -4 \beta_{y}\left[(1+\sqrt{2}) \beta_{x}-\sqrt{2} \beta_{y}\right]\left(a_{x} a_{x}^{+}\right)\left(a_{y} a_{y}^{+}\right) \\
& +\beta_{y}\left[\beta_{x}+2(1+\sqrt{2}) \beta_{y}\right]\left[e^{-2 i\left(\psi_{x}-\psi_{y}\right)}\left(a_{x}\right)^{2}\left(a_{y}^{+}\right)^{2}\right. \\
& \left.\left.+e^{2 i\left(\psi_{x}-\psi_{y}\right)}\left(a_{x}^{+}\right)^{2}\left(a_{y}\right)^{2}\right]\right\},
\end{aligned}
$$


TABLE III. The Lie factor $f_{4}$ for a family of sextupoles in the PEP-X achromat.

\begin{tabular}{lcc}
\hline \hline Coefficients $\left[\mathrm{m}^{-1}\right]$ & Analytical & Numerical \\
\hline$\left(a_{x} a_{x}^{+}\right)^{2}$ & $-6.1623 \times 10^{3}$ & $-6.0327 \times 10^{3}$ \\
$\left(a_{y} a_{y}^{+}\right)^{2}$ & $-3.6827 \times 10^{5}$ & $-3.6823 \times 10^{5}$ \\
$\left(a_{x} a_{x}^{+}\right)\left(a_{y} a_{y}^{+}\right)$ & $-4.2399 \times 10^{5}$ & $-4.2372 \times 10^{5}$ \\
$\left(a_{x}\right)^{2}\left(a_{y}^{+}\right)^{2}$ & $(-2.8032-3.8582 i) \times 10^{5}$ & $(-2.8059-3.8559 i) \times 10^{5}$ \\
$\left(a_{x}^{+}\right)^{2}\left(a_{y}\right)^{2}$ & $(-2.8032+3.8582 i) \times 10^{5}$ & $(-2.8059+3.8559 i) \times 10^{5}$ \\
\hline \hline
\end{tabular}

where $\psi_{x}$ and $\psi_{y}$ are the phase advances from the last sextupole to the end of the achromat. One can easily see, by using action-angle variables $a_{x}=\sqrt{J_{x}} e^{i \phi_{x}}, \quad a_{x}^{+}=$ $-i \sqrt{J_{x}} e^{-i \phi_{x}}, a_{y}=\sqrt{J_{y}} e^{i \phi_{y}}$, and $a_{y}^{+}=-i \sqrt{J_{y}} e^{-i \phi_{y}}$, that there are three tune shift terms and a single resonance driving term: $2 \nu_{x}-2 \nu_{y}$. The other resonances, $4 \nu_{x}, 4 \nu_{y}$, $2 \nu_{x}, 2 \nu_{y}$, and $2 \nu_{x}+2 \nu_{y}$, are canceled out among the eight sextupoles.

As an example, we took an arc of PEP-X and inserted eight sextupoles with a total integrated strength $S=$ $-80.0 \mathrm{~m}^{-2}$. The beta functions at the position of the sextupoles are $\beta_{x}=1.002 \mathrm{~m}$ and $\beta_{y}=7.746 \mathrm{~m}$, and the phase advances relative to the beginning of the cell are $\mu_{x}=2.0860 \mathrm{rad}$ and $\mu_{y}=1.6148 \mathrm{rad}$. All nonvanishing terms are calculated using two different methods and tabulated in Table III. As one can see in the table, the agreement between the analytical approach using Eq. (3.17) and numerical computation [12] based on the differential algebra is nearly perfect. To further illustrate the concept of cancellation, the fourth-order driving terms are plotted in Fig. 7 as a function of position in the achromat. As shown in the figure, the step changes occur at the locations of the sextupoles and the full cancellation occurs both at the midpoint and the end.

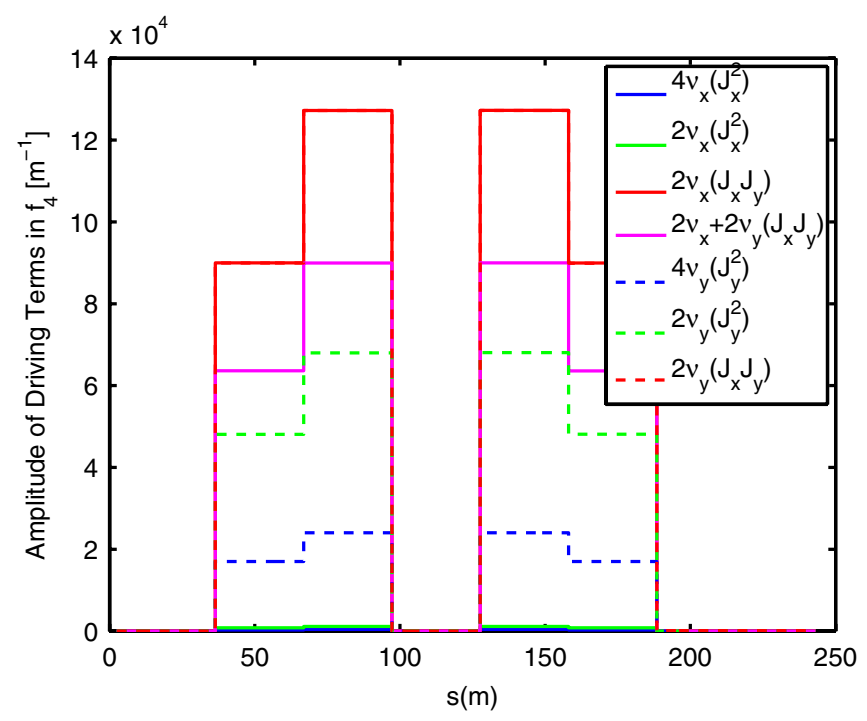

FIG. 7. The canceled fourth-order resonance terms driven by a family of the sextupoles as they accumulated in the PEP-X achromat.
This kind of cancellation in resonance effects within an achromat was discovered [12] first in one that consists of eight TME-type cells with phase advances of $\mu_{x}=3 \pi / 4$ and $\mu_{y}=\pi / 4$. Interestingly enough, in that case, the only nonvanishing resonance was $2 \nu_{x}+2 \nu_{y}$.

These cancellations of fourth-order resonances are extremely general. They only rely on the phase advances of the cell and its periodic property in the achromat. To remove the residual resonance $2 \nu_{x}-2 \nu_{y}$ and the three geometric aberrations, we need to utilize the specific structure of the PEP-X MBA cell, discussed in the next section. For multipole families of sextupoles, we have a similar result that will be presented in the Appendix.

\section{A fourth-order geometric achromat}

Recently, we used the lattice design code OPA [14] to optimize the settings of 10 sextupole families. Because of the cancellations of most resonances, we only needed to control the nonlinear chromaticities up to the second order, the three remaining amplitude-dependent tune shifts, and the residual $2 \nu_{x}-2 \nu_{y}$ resonance. A good solution with small nonlinearities was found [11]. The derivatives of the betatron tunes are calculated using the normal form method [28] and tabulated in Table IV along with a new solution based on 4 th-order achromats $\left(f_{3}=f_{4}=0\right)$. As one can see from the table, the tune shift terms are significantly reduced using the 4th-order achromats without any degradation in the chromatic parts. The small residuals are due to the nonlinear kinematic terms in the Hamiltonian.

As outlined in the previous section, one can compute all contributions to $f_{4}$ from all families of sextupoles by using Eqs. (3.17) and (A1). To find a 4th-order geometric achromat, we simply adjusted the strengths of six families of the harmonic sextupoles to eliminate the five nonvanishing

TABLE IV. The nonlinear chromaticities and tune shifts due to betatron amplitudes in the PEP-X ultimate storage ring.

\begin{tabular}{lcc}
\hline \hline Derivatives of tunes & OPA solution & 4th-order achromats \\
\hline$\partial \nu_{x, y} / \partial \delta$ & 0,0 & 0,0 \\
$\partial^{2} \nu_{x, y} / \partial \delta^{2}$ & $-54,-95$ & $-57,-89$ \\
$\partial^{3} \nu_{x, y} / \partial \delta^{3}$ & $+1350,-104$ & $+1332,-150$ \\
$\partial \nu_{x} / \partial J_{x}\left[\mathrm{~m}^{-1}\right]$ & -5354 & +253 \\
$\partial \nu_{x, y} / \partial J_{y, x}\left[\mathrm{~m}^{-1}\right]$ & +19610 & +1158 \\
$\partial \nu_{y} / \partial J_{y}\left[\mathrm{~m}^{-1}\right]$ & -76390 & -228 \\
\hline \hline
\end{tabular}



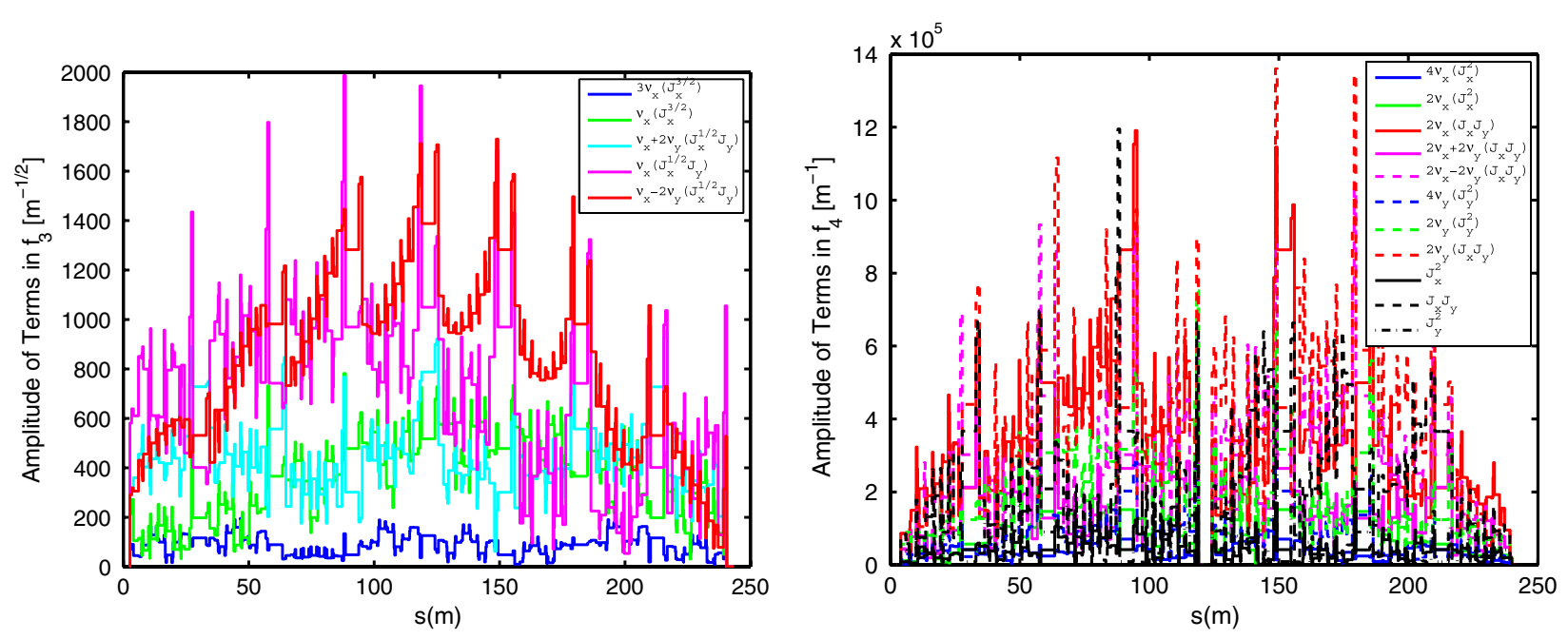

FIG. 8. All 3rd-order resonance terms in $f_{3}$ (left) and all 4th-order resonance as well as three tune shift terms in $f_{4}$ (right) generated by the 10 families of sextupoles as they accumulated in the PEP-X achromat.

terms in $f_{4}$. In fact, we have an infinite set of solutions which can be found by searching numerically using the Nelder-Mead method. We use the extra degree of freedom to minimize the peak value of the harmonic sextupoles. As a result, the peak strength is reduced by $25 \%$ from the OPA solution. All terms in $f_{3}$ and $f_{4}$ are plotted as a function of the position along one of the arcs in PEP-X in Fig. 8. One can see from the figures that the numerical calculations using the actual beam line confirm the analytical results. Although we have not analyzed the chromatic effects in this paper, in fact both the first- and second-order dispersions are also canceled within this achromat as shown in Ref. [13].

For a comparison with OPA, it is worth pointing out what OPA does: it uses a gradient search minimizer with empirically set weight factors on analytic formulas for the geometric Hamiltonian modes of 1 st and 2 nd order in sextupole strength and on chromaticities up to 3 rd order obtained by numeric differentiation. So it may be rather blind and could end up in some local minimum depending on starting values and the user's selection of weight factors. To set $f_{3}=f_{4}=0$ in our method may be a superior approach leading to a true optimum.

It is worth noting that the theory of higher-order achromats was developed by Dragt [29] who found also a numerical example of 4th-order achromat using not only sextupoles but also octupoles. Comparing to his solution, ours is much simpler and more practical because octupoles are not used.

\section{E. Damping wiggler}

Because the emittance of PEP-X will increase by a factor of 2 from the zero-current natural value of $29 \mathrm{pm}$ rad achieved with the 7BA lattice due to intrabeam scattering with 200-mA stored beam current (Sec. V), a further reduction in emittance by about a factor of 3 is needed to reach the diffraction limit for $1-\AA \mathrm{x}$ rays (assuming $100 \%$ horizontal-vertical emittance coupling). This emittance reduction can be achieved using one or more strong damping wigglers in one or more dispersion-free regions.

The relative reduction in natural emittance from $\epsilon_{0}$ to $\epsilon_{w 0}$ caused by a damping wiggler in PEP-X can be estimated using an approximate analytical expression [30]:

$$
\frac{\epsilon_{0 w}}{\epsilon_{0}}=\left(\frac{I_{x 0}}{I_{x w}}\right) \frac{1+\frac{4 C_{q}}{15 \pi I_{x 0}} N_{p} \gamma^{2} \frac{\left\langle\beta_{x w}\right\rangle \rho_{o}}{\epsilon_{x 0} \rho_{w}^{2}} \theta_{w}^{3}}{1+\frac{1}{2} N_{p} \frac{\rho_{o}}{\rho_{w}} \theta_{w}},
$$

where $I_{x w}, I_{x 0}$ are damping partition numbers with and without wigglers, $N_{p}$ is the number of wiggler periods, $\left\langle\beta_{x w}\right\rangle$ the average horizontal $\beta$ function in the wiggler, $\rho_{w}$ the bending radius at peak wiggler field, $\theta_{w}=\lambda_{w} / 2 \pi \rho_{w}$, and $\lambda_{w}$ the wiggler period length. Here $\rho_{o}=\rho_{c} \rho_{m} /$ $\left[r \rho_{c}+(1-r) \rho_{m}\right]$ is the effective bending radius in the MBA cell, with $\rho_{c}$ and $\rho_{m}$ being the bending radii in the main cell dipole and the matching dipole, respectively, and $r$ being the relative contribution from the matching dipoles to the total bending angle.

It follows that the emittance reduction depends on the wiggler period length, the wiggler peak field, and the total wiggler length. Using Eq. (3.18), Fig. 9 shows the ratio of $\epsilon_{0 w} / \epsilon_{0}$ versus the wiggler peak field and the total wiggler length for various values of wiggler period length, where the wiggler is inserted in a long straight section with $\left\langle\beta_{x}\right\rangle=12.4 \mathrm{~m}$. One can see that most of the damping occurs within $100 \mathrm{~m}$ of the wiggler length, and that a wiggler period below $5 \mathrm{~cm}$ does not significantly improve the damping. Selecting a $90-\mathrm{m}$ long wiggler with a $5-\mathrm{cm}$ period, it follows that the optimal peak field is $1.5 \mathrm{~T}$. It should be noted that a short $5-\mathrm{cm}$ wiggler period implies a small wiggler gap of $7.7 \mathrm{~mm}$ when using a hybrid magnet design [31]. A wiggler with the above parameters has been modeled in the lattice using an array of alternating field 

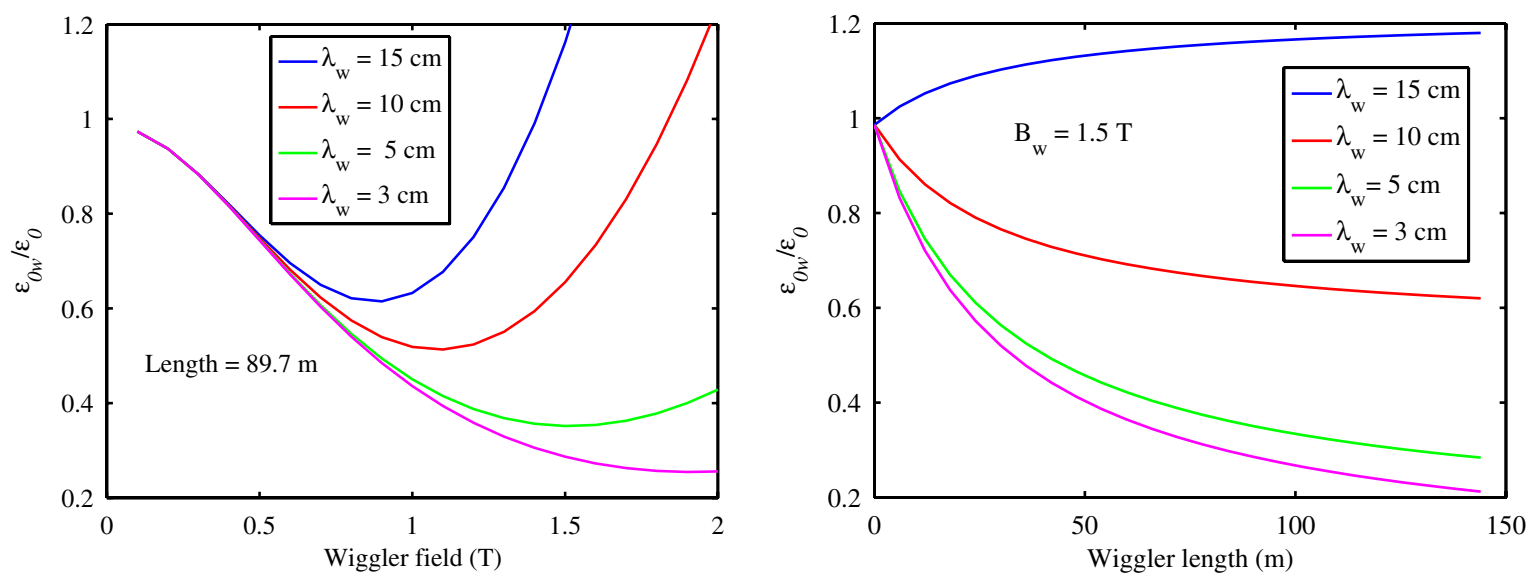

FIG. 9. Relative emittance reduction versus wiggler field (left) and versus wiggler length (right) for various values of wiggler period.

short dipoles. The wiggler is placed in one $123-\mathrm{m}$ long FODO straight section, where it is split into 18 sections to fit between the quadrupoles. The resultant emittance with wigglers at zero current is $\epsilon_{0 w}=11 \mathrm{pm}$-rad.

The damping wiggler creates various negative effects on the PEP-X beam. It increases the beam rms energy spread from $0.072 \%$ to $0.12 \%$, and the radiation loss per turn from 0.36 to $2.95 \mathrm{MeV}$. The latter amounts to $0.59 \mathrm{MW}$ at a $200 \mathrm{~mA}$ current. Finally, the wiggler field has intrinsic nonlinear components on the beam trajectory affecting large amplitude particles.

\section{DYNAMIC APERTURE}

Ultimately, the goal of minimizing sextupole nonlinear aberrations is to maximize the PEP-X dynamic aperture for efficient horizontal injection and long beam lifetime. The two sextupole solutions described in Sec. III E were compared: the empirical optimization using OPA and the analytical 4th-order geometric achromat. Note that the latter uses the same chromatic sextupole strengths as in the OPA solution. The dynamic aperture was obtained in particle tracking simulations using the LEGO [32] and ELEGANT [33] codes. The calculations included on-momentum and off-momentum particles and magnet errors. To include the effects of the wiggler intrinsic nonlinear field, the 1st order dipole wiggler model was replaced by an exact nonlinear field wiggler model [34] in the LEGO simulations.

\section{A. Error-free aperture}

To verify the effect of residual sextupole aberrations, dynamic apertures for the OPA and the 4th-order geometric achromat solutions were compared without magnet errors and with momentum error $\delta$ up to 2\%, as shown in Fig. 10. The better cancellation of sextupole 4 th-order geometric terms in the achromat solution results in a superior onmomentum aperture in both $x$ and $y$ planes as compared to the OPA solution. The achromat off-momentum aperture is also slightly better, but much closer to the OPA aperture due to the fact that the two solutions use the same strengths for the chromatic sextupoles, leading to similar chromatic
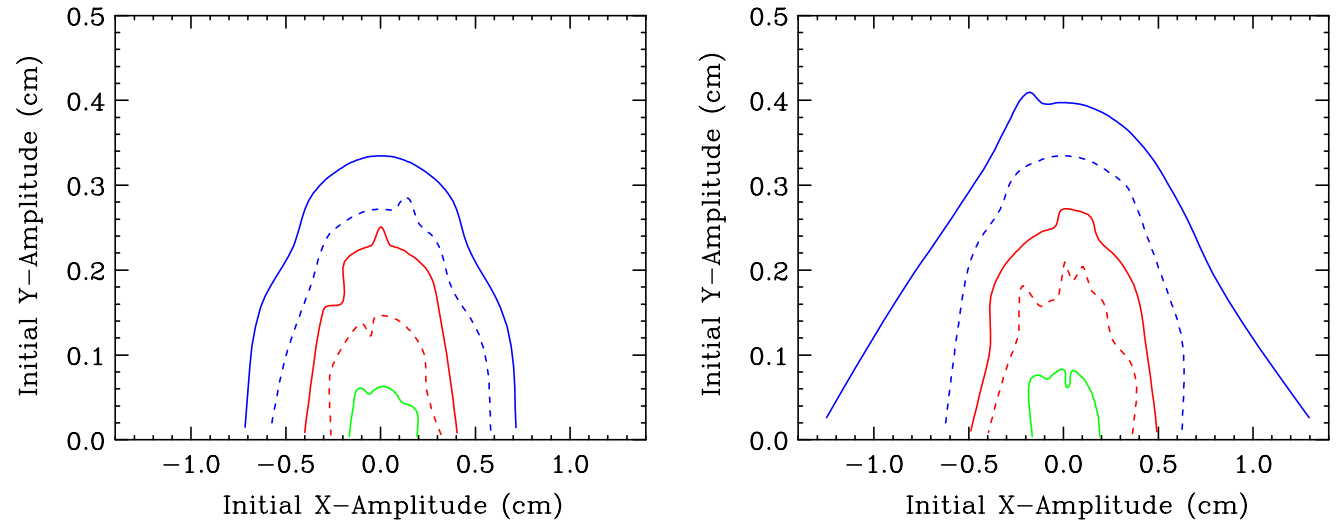

FIG. 10. Momentum dependent dynamic aperture without errors for OPA (left) and 4th-order geometric achromat (right) solutions with chromaticity set to zero, where $\delta=0$ (blue solid), $0.5 \%$ (blue dashed), $1 \%$ (red solid), $1.5 \%$ (red dashed), and $2 \%$ (green). 

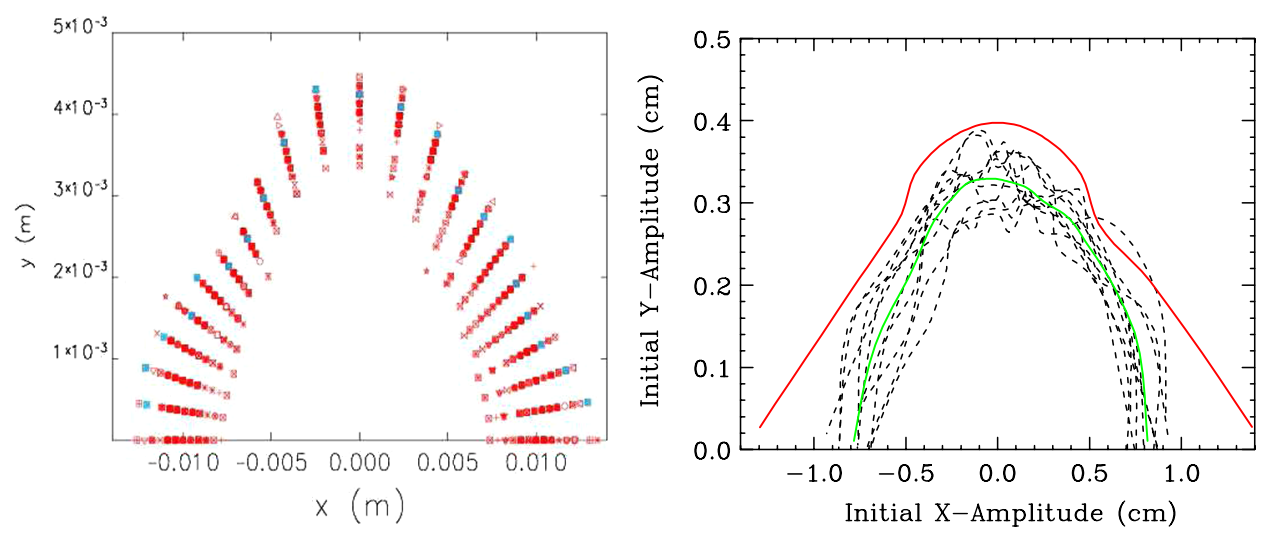

FIG. 11. Dynamic apertures in ELEGANT (left) simulation for 50 sets of random errors (red) and without errors (black) and in LEGO (right) for 10 sets of random errors (dash), the average aperture (green), and aperture without errors (red).

aberrations (see Table IV). Based on this comparison, we chose the achromat solution for the PEP-X ultimate lattice.

\section{B. Error tolerances}

Magnet field and alignment errors create linear and nonlinear optics perturbations. These include distortion of the closed orbit and betatron functions, transverse coupling, chromaticity, variation of betatron tune with amplitude, and excitation of betatron resonances leading to reduced dynamic aperture. To maintain a sufficient aperture, the ring must include efficient correction schemes; also, the magnitude of such errors must be limited to an acceptable level. To estimate the error sensitivities for the PEP-X lattice with the 4th-order achromat sextupole solution, LEGO and ELEGANT tracking simulations were performed.

The ELEGANT simulations did not include actual beam correction. Instead, it was assumed that the errors are already corrected, and that the residual effects are $1 \%$ beta beat and $1 \%$ transverse coupling. These were simulated with sufficiently small random quadrupole field and tilt errors. The resultant dynamic aperture for 50 machine settings of random errors is shown in Fig. 11.

LEGO simulations included realistic correction of orbit, beta beat, linear chromaticity, transverse coupling, and vertical dispersion. The studied errors included magnet field errors, horizontal and vertical misalignment, magnet roll errors, and higher-order multipole field errors. The latter were based on the measured field in the PEP-II magnets [35] but applied to a smaller bore radius of the PEP-X magnets. No errors were applied to beam position monitors, and the linear chromaticity was adjusted to +1 .

It was found that rms misalignment of $20 \mu \mathrm{m}$, rms roll error of $0.1 \mathrm{mrad}$, and relative rms field error of $10^{-3}$ in dipoles, quadrupoles, and sextupoles are acceptable. Dynamic apertures with the above errors including the high order multipole field errors for 10 random settings after correction are shown in Fig. 11. Here, the average onmomentum horizontal dynamic aperture is $8 \mathrm{~mm}$ which is sufficient for off-axis injection assuming a high quality injected beam with $1 \mu \mathrm{m}$-rad normalized emittance and effective septum width of $3 \mathrm{~mm}$ [19]. It should be noted that a smaller dynamic aperture could still be accommodated using on-axis injection.

\section{IBS AND TOUSCHEK LIFETIME}

Intrabeam scattering (IBS) describes multiple Coulomb scattering that leads to growth in emittance and energy spread in electron machines, whereas the Touschek effect concerns large single Coulomb scattering events where energy transfer from transverse to longitudinal planes leads to particle loss. In low emittance machines such as PEP-X, both effects are important.

\section{A. Intrabeam scattering}

We understand that obtaining round beams in a storage ring will likely entail the use of coupling or vertical dispersion. But for simplicity here, for the purpose of IBS calculations, we assume that the vertical emittance is primarily generated by the coupling, and the effects of the vertical dispersion can be ignored. Then the vertical emittance is proportional to the horizontal emittance, and we write

$$
\epsilon_{x}=\frac{\epsilon}{1+\kappa} \quad \text { and } \quad \epsilon_{y}=\frac{\kappa \epsilon}{1+\kappa}
$$

with $\kappa$ being the coupling constant between 0 and 1 and $\epsilon=\epsilon_{x}+\epsilon_{y}$ being the sum emittance at finite current with IBS. The nominal (no IBS) horizontal and vertical emittances are given by $\epsilon_{x 0}=\epsilon_{0 w} /(1+\kappa)$ and $\epsilon_{y 0}=$ $\kappa \epsilon_{0 w} /(1+\kappa)$, where $\epsilon_{0 w}$ is the natural emittance with the damping wigglers at zero current and its value is given in Table II. Note that our treatment of coupling is a simplified one that is often used in the case of small coupling; it assumes that the eigenfunctions of the transverse motion are only slightly perturbed by the coupling. In our case of full coupling we need to make a similar 
assumption. In any case, for the PEP-X IBS calculations to be presented below, we will at the end confirm the results with calculations using the program SAD [36], one that treats coupling correctly without assumptions.

In our calculation here we make the assumption that the transverse IBS growth rate approximately satisfies

$$
\frac{\epsilon_{x 0}}{\tau_{x}}+\frac{\epsilon_{y 0}}{\tau_{y}}-\frac{\epsilon_{x}}{\tau_{x}}-\frac{\epsilon_{y}}{\tau_{y}}+\frac{\epsilon_{x}}{T_{x}}=0,
$$

where $\tau_{x}, \tau_{y}$, signify the radiation damping times in $x, y$, and $1 / T_{x}$ gives the IBS growth rate in amplitude (the growth rate in emittance is just $2 / T_{x}$ ). A similar expression is also used in ELEGANT. The first two terms in Eq. (5.2) represent quantum excitation growth rates, the next two terms those of radiation damping, and the last term that of IBS. (A similar equation applies for the growth in momentum.) Writing Eq. (5.2) in terms of the sum emittances $\epsilon$ and $\epsilon_{x w}$, and solving the corresponding energy spread equation, we find that the steady-state values are given by

$$
\epsilon=\frac{\epsilon_{0 w}}{1-\tau_{x}^{*} / T_{x}} \quad \text { and } \quad \sigma_{\delta}^{2}=\frac{\sigma_{\delta w}^{2}}{1-\tau_{s} / T_{p}},
$$

where $\tau_{x}^{*}=\tau_{x} /\left(1+\kappa \tau_{x} / \tau_{y}\right)$. The quantities $\sigma_{\delta w}, \tau_{s}$, and $1 / T_{p}$ signify, respectively, the nominal beam size, the radiation damping time, and the IBS growth rate in momentum. Note that taking $\tau_{x}^{*}=\tau_{x}$ does not significantly affect the results in the case of small coupling; in the case $\kappa=1$, however, it overestimates the effective IBS growth rate by a factor $\sim 2$ (if $\tau_{x} \sim \tau_{y}$ ).

For PEP-X IBS growth rates we employ the BjorkenMtingwa (B-M) formulation [37], using the Nagaitsev [38] algorithm for efficient calculation. B-M gives the local growth rates $\delta\left(1 / T_{x}\right)$ and $\delta\left(1 / T_{p}\right)$ as integrals that depend on beam properties and local lattice properties. These integrals are numerically performed for all positions around the ring, and then the growth rates are averaged to give $\left\langle\delta\left(1 / T_{x}\right)\right\rangle=1 / T_{x},\left\langle\delta\left(1 / T_{p}\right)\right\rangle=1 / T_{p}$, where \langle\rangle means to average around the ring. Given the growth rates, the steady-state $\epsilon$ and $\sigma_{\delta}$ are obtained by solving Eqs. (5.3) simultaneously. Since the growth rates depend on the beam emittances, energy spread, and bunch length, Eqs. (5.3) are solved by iteration using a Newton's method.

A simplified model of the B-M equations that can be used (with slight modification) to approximate the results for PEP-X is the so-called "high energy approximation" [39]. We present it here since it relatively clearly shows the parameter dependence of IBS, though to obtain the numerical results for PEP-X (given below) we will use the more accurate B-M equations. According to this simplified model the IBS growth rate in energy spread is given by

$$
\frac{1}{T_{p}} \approx \frac{r_{e}^{2} c N_{b}(\log )}{16 \gamma^{3} \epsilon_{x}^{3 / 4} \epsilon_{y}^{3 / 4} \sigma_{z} \sigma_{\delta}^{3}}\left\langle\sigma_{H} g(a / b)\left(\beta_{x} \beta_{y}\right)^{-1 / 4}\right\rangle .
$$

Here $r_{e}$ is the classical radius of the electron, $c$ the speed of light, $N_{b}$ the number of electrons per bunch, $(\log )$ the Coulomb $\log$ factor, $\gamma$ the Lorentz energy factor, $\sigma_{z}$ the bunch length, and $\beta_{x}$ and $\beta_{y}$ the optical beta functions. Other factors in Eq. (5.4) are defined by

$$
\begin{gathered}
\frac{1}{\sigma_{H}^{2}}=\frac{1}{\sigma_{\delta}^{2}}+\frac{\mathcal{H}_{x}}{\epsilon_{x}}, \quad a=\frac{\sigma_{H}}{\gamma} \sqrt{\frac{\beta_{x}}{\epsilon_{x}}}, \quad b=\frac{\sigma_{H}}{\gamma} \sqrt{\frac{\beta_{y}}{\epsilon_{y}}}, \\
g(\alpha)=\alpha^{(0.021-0.044 \ln \alpha)},
\end{gathered}
$$

with $\mathcal{H}_{x}$ the dispersion invariant defined in Eq. (3.3). Note that the high energy approximation has validity when $a, b \ll 1$, which holds for PEP-X parameters.

In the high energy approximation, the horizontal IBS growth rate is given in terms of the momentum growth rate simply as

$$
\frac{1}{T_{x}}=\frac{\sigma_{\delta}^{2}}{\epsilon_{x}}\left\langle\mathcal{H}_{x} \delta\left(1 / T_{p}\right)\right\rangle
$$

We see that only the arcs contribute significantly to IBS-induced emittance growth, since only in the arcs is $\mathcal{H}_{x}$ nonzero. Note that in the original description of the high energy approximation (Ref. [39]), $\left\langle\mathcal{H}_{x} \delta\left(1 / T_{p}\right)\right\rangle$ in Eq. (5.7) is replaced by $\left\langle\mathcal{H}_{x}\right\rangle\left(1 / T_{p}\right)$; for the PEP-X lattice, however, there are correlations that make that version of the equation a poor approximation to B-M.

In scattering calculations like IBS, a Coulomb log term, ( $\log$ ) in Eq. (5.4), is often used to take into account the contribution of very large and very small impact parameter events. Because of the small impact parameter events, the tails of the steady-state bunch distributions are not Gaussian and the standard way of computing (log) overemphasizes their importance. To better represent the size of the bunch core, we adjust (log) to cut away events with growth rate greater than the synchrotron damping rate, as was first proposed by Raubenheimer [40,41]. For PEP-X, $(\log )$ becomes $\approx 11$.

For our IBS calculations for PEP-X using lattice parameters found in Table II, we assume that the nominal current is below the threshold of the microwave instability and that potential well bunch lengthening is not significant (so that $\sigma_{z}=\sigma_{z w} \sigma_{\delta} / \sigma_{\delta w}$ ). We assume the vertical emittance is given by coupling with $\kappa=1$. The results of our IBS calculations for the PEP-X lattice at the nominal $I=$ $200 \mathrm{~mA}$ with the number of bunches $M=3300$, which corresponds to $0.5 \mathrm{nC}$ bunch charge, are shown in Table $\mathrm{V}$, where we give steady-state emittances, $\epsilon_{x}$ and $\epsilon_{y}$, energy spread $\sigma_{\delta}$, and bunch length $\sigma_{z}$. We note that for PEP-X, IBS has little effect on $\sigma_{\delta}$ and $\sigma_{z}$; however, at the nominal current $\epsilon_{x}$ is double the zero-current value.

At nominal current the horizontal IBS growth rate is $T_{x}^{-1}=52 \mathrm{~s}^{-1}$, and the energy growth rate $T_{p}^{-1}=7.4 \mathrm{~s}^{-1}$. The growth rate, as expected, is significant only in the arcs. Note that from the high energy approximation, Eqs. (5.4) 
TABLE V. Steady-state beam parameters with $200 \mathrm{~mA}$ in PEP-X and $x-y$ coupling parameter $\kappa=1$ : nominal (zerocurrent) horizontal $\epsilon_{x 0}$ and vertical $\epsilon_{y 0}$ emittances; steady-state horizontal $\epsilon_{x}$ and vertical $\epsilon_{y}$ emittances; relative energy spread $\sigma_{\delta}$, bunch length $\sigma_{z}$; and Touschek lifetime $\mathcal{T}$.

$\underline{\epsilon_{x 0}[\mathrm{pm}] \epsilon_{y 0}[\mathrm{pm}] \epsilon_{x}[\mathrm{pm}] \epsilon_{y}[\mathrm{pm}] \sigma_{\delta}\left[10^{-3}\right] \sigma_{z}[\mathrm{~mm}] \mathcal{T}[\mathrm{hrs}]}$

\begin{tabular}{lllllll}
5.5 & 5.5 & 11.5 & 11.5 & 1.25 & 3.12 & 3.5 \\
\hline
\end{tabular}

and (5.7), we obtain $T_{x}^{-1}=53.7 \mathrm{~s}^{-1}$ and $T_{p}^{-1}=8.9 \mathrm{~s}^{-1}$, in reasonable agreement to the Bjorken-Mtingwa solution.

A comparison IBS calculation was performed using the optics program SAD [36]. SAD treats coupling without assumptions by obtaining, e.g., the true emittance invariants, and it can also solve the B-M IBS equations. For the calculation in the dispersion-free regions of PEP-X, we first adjusted quadrupole strengths to bring the tunes close together. Then 400 quadrupole magnets (in these regions) were rotated by small random amounts and adjusted by an overall scale factor to give $\epsilon_{x 0} \approx \epsilon_{y 0}$. Finally IBS calculations were performed, giving $\epsilon_{x} \approx \epsilon_{y} \approx 11 \mathrm{pm}$, a result not far from our earlier obtained $11.5 \mathrm{pm}$. Note that the SAD calculations were repeated for 10 seeds (for the random number generator), and the results varied by only a few percent.

In addition to the calculations for nominal current, we have also calculated the steady-state emittances $\epsilon_{x}$ and $\epsilon_{y}$ as functions of beam current $I$; the result is shown in Fig. 12 (the solid curve). In our calculations we have again observed that for PEP-X the growth of longitudinal emittance due to IBS is very small. This means that, to a good approximation, $\sigma_{\delta}$ and $\sigma_{z}$ can be taken to have their nominal values and one need only solve the first of Eqs. (5.3). In this case the horizontal emittance as a function of current can be approximated by a solution (the maximum real solution) of the equation

$$
\left(\frac{\epsilon_{x}}{\epsilon_{x 0}}\right)^{5 / 2}-\left(\frac{\epsilon_{x}}{\epsilon_{x 0}}\right)^{3 / 2}=\alpha\left(\frac{I}{I_{A}}\right)
$$

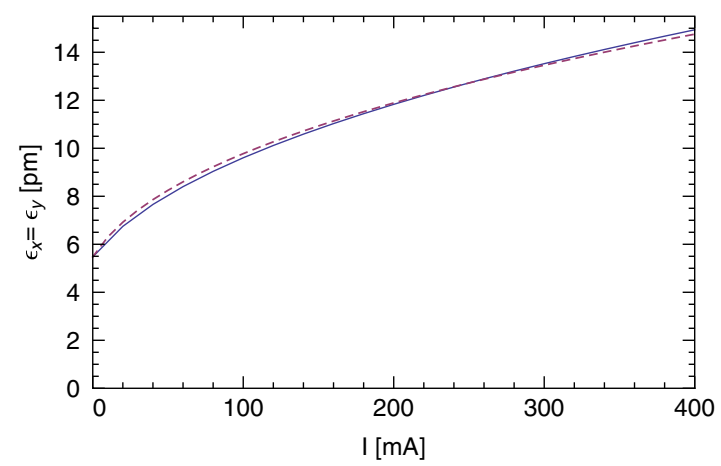

FIG. 12. Steady-state emittances as a function of bunch current in PEP-X with round beams $(k=1)$.

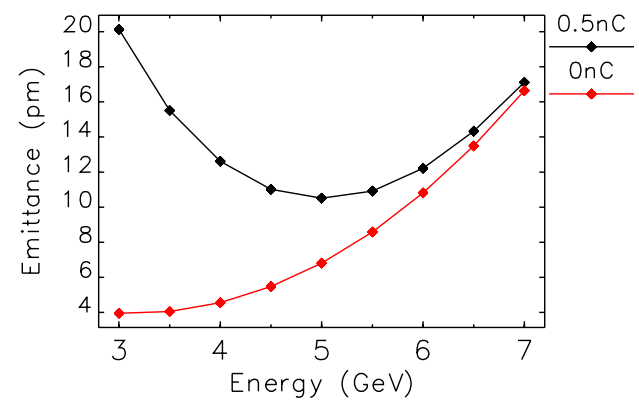

FIG. 13. Emittance $\epsilon_{x}=\epsilon_{y}$ vs energy for a round beam at nominal bunch current (black) and at zero current (red).

with $\alpha$ a constant and $I_{A}=17 \mathrm{kA}$ the Alfvén current. Here the best fit is obtained with $\alpha=3.2 \times 10^{5}$ (see the dashed curve in Fig. 12).

Finally, to demonstrate that $4.5 \mathrm{GeV}$ is near the optimal energy for our lattice, we have performed IBS calculations for different energies. Note that the lattice is scalable with energy except in the wiggler and undulator regions which are assumed to have nominally fixed-field magnets. In Fig. 13 we plot emittance $\epsilon_{x}=\epsilon_{y}$ vs electron energy $E$; we see that the emittance minimum is broad, and that the minimum is near our nominal energy.

\section{B. Touschek lifetime}

Touschek lifetime calculations normally follow the flatbeam equation of Brück [42], with modifications by Piwinski [43]. For round beam calculations we will begin here with the more general formula (i.e. not limited to flat beams) due to Piwinski $[43,44]$. With the Touschek effect the number of particles in a bunch decays with time $t$ as

$$
N_{b}=\frac{N_{b 0}}{1+t / \mathcal{T}}
$$

with $N_{b 0}$ the initial bunch population, and $\mathcal{T}$ the Touschek lifetime. Note that the decay is not exponential. The lifetime is given by [43]

$$
\frac{1}{\mathcal{T}}=\frac{r_{e}^{2} c N_{b}}{8 \sqrt{\pi} \beta^{2} \gamma^{4} \sigma_{z} \sigma_{\delta} \epsilon_{x} \epsilon_{y}}\left\langle\sigma_{H} \mathcal{F}\left(\delta_{m}\right)\right\rangle
$$

with

$$
\begin{gathered}
\mathcal{F}\left(\delta_{m}\right)=\int_{\delta_{m}^{2}}^{\infty} \frac{d \tau}{\tau^{3 / 2}} e^{-\tau B_{+}} I_{0}\left(\tau B_{-}\right)\left[\frac{\tau}{\delta_{m}^{2}}-1-\frac{1}{2} \ln \left(\frac{\tau}{\delta_{m}^{2}}\right)\right], \\
B_{ \pm}=\frac{1}{2 \beta^{2} \gamma^{2}}\left|\frac{\beta_{x} \sigma_{x}^{2}}{\epsilon_{x} \tilde{\sigma}_{x}^{2}} \pm \frac{\beta_{y}}{\epsilon_{y}}\right|
\end{gathered}
$$

where $\sigma_{H}$ is defined in Eq. (5.5), and again \langle\rangle indicates averaging around the ring. In this formula the only assumptions are that there is no vertical dispersion and that the energies are nonrelativistic in the beam rest frame 


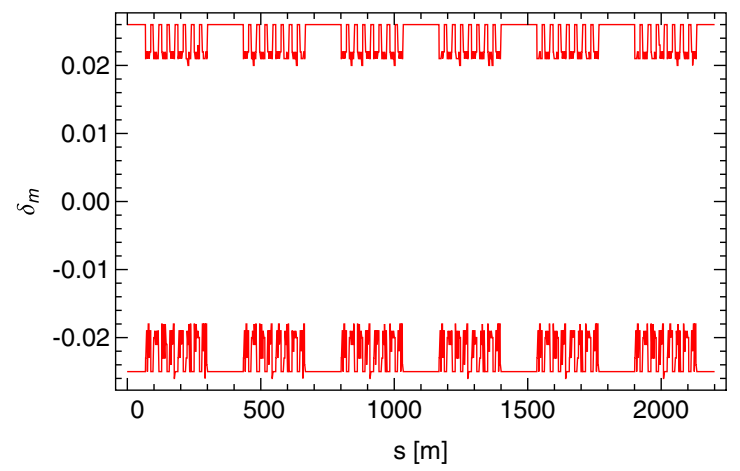

FIG. 14. The momentum acceptance, $\delta_{m}$, for PEP-X with machine errors. This function is used in finding the Touschek lifetime.

$\left(\gamma^{2} \sigma_{x}^{2} / \beta_{x}^{2}, \gamma^{2} \sigma_{y}^{2} / \beta_{y}^{2} \ll 1\right)$; there is no requirement that the beam be flat. Parameters are average velocity over the speed of light $\beta$, modified Bessel function of the first kind $I_{0}$, relative momentum acceptance $\delta_{m}$ (half aperture), and beam sizes $\sigma_{x}=\sqrt{\beta_{x} \epsilon_{x}+\eta_{x}^{2} \sigma_{\delta}^{2}}$ and $\tilde{\sigma}_{x}=\sqrt{\beta_{x} \epsilon_{x}+\beta_{x} \mathcal{H}_{x} \sigma_{\delta}^{2}}$.

We have calculated the momentum aperture as a function of location in PEP-X, including machine errors described in Sec. IV B, in the following manner. In tracking, at a given position $s$, a beam particle is given an initial relative positive momentum kick $\delta_{m}$, and it undergoes betatron and synchrotron oscillations. The largest value of $\delta_{m}$ for which the particle survives defines the positive momentum aperture at position $s$. Then the same is done for a negative momentum kick. The results are displayed in Fig. 14. This local momentum acceptance is used in Touschek lifetime calculation for PEP-X and the result is 3.5 hours. Note that the calculation without errors yields 11 hours.

Finally, to study the sensitivity of Touschek lifetime to momentum acceptance, we computed $\mathcal{T}$ vs the global momentum acceptance parameter, $\delta_{m}$ (see Fig. 15, blue symbols). The dashed curve gives the fit: $\mathcal{T}=$ $0.088\left(\delta_{m} / 0.01\right)^{5}$ hrs. We see that if we want a reasonable lifetime, we cannot allow $\delta_{m}$ to be significantly worse than \pm 0.02 .

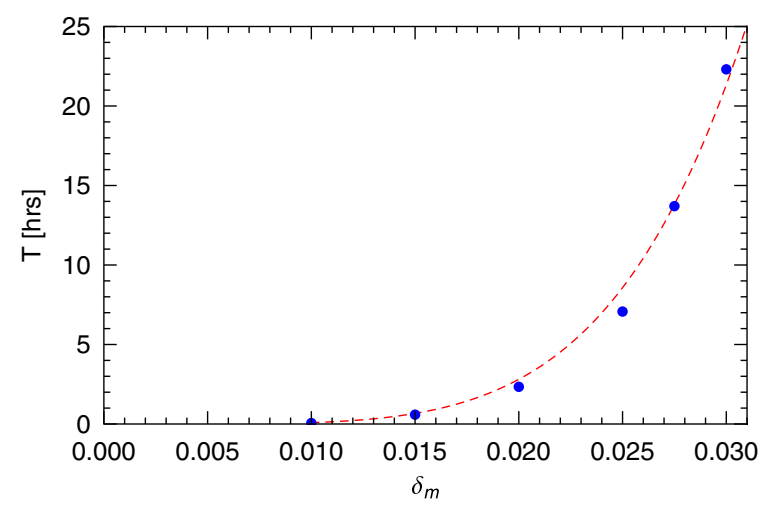

FIG. 15. Touschek lifetime $\mathcal{T}$ vs (global) momentum acceptance parameter, $\delta_{m}$ (blue symbols). The dashed curve gives the fit: $T=0.088\left(\delta_{m} / 0.01\right)^{5}$ hrs.

\section{IMPEDANCE AND INSTABILITIES}

For the baseline design of PEP-X [19], an impedance budget was accumulated and calculations were performed on longitudinal and transverse instability thresholds and on growth rates. In the present report we again perform such calculations but go into less detail. We justify this by the fact that the present bunch current is a factor of 7.5 smaller than the previous one, and consequently instabilities are not such an important issue. We here briefly address three instabilities: (i) the single-bunch microwave instability excited by coherent synchrotron radiation (CSR), (ii) the single-bunch transverse mode coupling instability due to the resistance in the walls, and (iii) the multibunch transverse instability driven by the wall resistance.

\section{A. Microwave instability due to CSR}

For the baseline design of PEP-X an impedance budget and single-bunch wake representing the entire ring was generated. These were used to estimate the threshold of the microwave instability, which was found to be comfortably above the earlier design current of 1.5 A. Here we estimate the microwave threshold due only to one contributor to the impedance, shielded CSR. In the model used for the calculations the beam is assumed to be moving in a circle of radius $\rho$ (in the plane $y=0$ ) between two parallel plates at locations $y= \pm h$. In normalized units the threshold current $S$ th is given as a function of shielding parameter $\Pi$ by [45]

$$
S^{\text {th }}=0.50+0.12 \Pi \text {, }
$$

with

$$
S=\frac{e N_{b} \rho^{1 / 3}}{2 \pi \nu_{s} \gamma \sigma_{\delta} \sigma_{z}^{4 / 3}}, \quad \Pi=\frac{\sigma_{z} \rho^{1 / 2}}{h^{3 / 2}} .
$$

with $N_{b}$ being the number of electrons per bunch and $\nu_{s}$ being the synchrotron tune.

The PEP-X vacuum chamber in the arcs is elliptical with axes $\left(b_{x}, b_{y}\right)=(20.0,12.5) \mathrm{mm}$ and bending radius $\rho=$ $100.8 \mathrm{~m}$; we let $h=12.5 \mathrm{~mm}$ in the calculations. With these assumptions we find that for PEP-X the shielding is significant: with $\Pi=22.7$, the threshold bunch population $N_{b}^{\text {th }}=4.9 \times 10^{10}$, and the threshold current $I^{\text {th }}=3.6 \mathrm{~A}-$ high above the design current. (Note that, even if we were to increase the aperture so that there is no shielding, $S^{\text {th }}=$ 0.50 and the threshold would be $0.58 \mathrm{~A}$, significantly above the design current.)

\section{B. Transverse single-bunch instability}

In most light sources with regions of small-aperture vacuum chambers, the resistive wall is the dominant contribution to the transverse single-bunch instability. The kick factor (the average kick experienced over a bunch) for a Gaussian bunch passing through a round, resistive beam pipe is given by 
TABLE VI. PEP-X beam pipe chamber types, giving total length, cross-sectional shape, half-height in $x$ and $y$, type of metal coating, and average beta function. Note that straights are divided into regular $(r)$ and injection $(i)$ types.

\begin{tabular}{lccccc}
\hline \hline Type & Length $[\mathrm{m}]$ & Shape & $\left(b_{x}, b_{y}\right)[\mathrm{mm}]$ & $\operatorname{Metal}\left\langle\beta_{y}\right\rangle[\mathrm{m}]$ \\
\hline Arcs & 1318 & Elliptical & $(20.0,12.5)$ & $\mathrm{Al}$ & 7.0 \\
Straights $r$ & 510 & Round & $(48.0,48.0)$ & $\mathrm{Al}$ & 15.6 \\
Straights $i$ & 123 & Round & $(48.0,48.0)$ & $\mathrm{Al}$ & 60.0 \\
Undulators & 158 & Elliptical & $(20.0,3.0)$ & $\mathrm{Cu}$ & 2.8 \\
Wigglers & 90 & Rectangular & $(22.5,4.0)$ & $\mathrm{Cu}$ & 12.0 \\
\hline \hline
\end{tabular}

$$
\kappa_{y}=(0.723) \frac{c}{\pi^{3 / 2} b^{3}} \sqrt{\frac{Z_{0}}{\sigma_{z} \sigma_{c}}},
$$

with $b$ the radius of the pipe, $Z_{0}=377 \Omega, \sigma_{z}$ the bunch length, and $\sigma_{c}$ the conductivity of the beam pipe. The single-bunch threshold current is given by [46]

$$
I_{b}^{\mathrm{th}} \approx 0.7 \frac{4 \pi c \nu_{s}(E / e)}{\mathcal{C}} \frac{1}{\sum_{i} \ell_{i} \beta_{y, i} \kappa_{y, i}},
$$

with $\mathcal{C}$ the circumference of the ring. The multibunch threshold is $I^{\text {th }}=M I_{b}^{\text {th }}$, with $M$ the number of bunches. Equation (6.4) allows for several region types in the ring, each of total length $\ell$, beta function $\beta_{y}$, and kick factor $\kappa_{y}$.

The five region types of PEP-X and their beam pipes are described in Table VI. For the threshold calculation we use the information in the table, letting the vertical half aperture be $b$; the conductivities of $\mathrm{Al}(\mathrm{Cu})$ is taken to be $3.5(5.9) \times 10^{7} \Omega^{-1} \mathrm{~m}^{-1}$. We see that the undulator and wiggler sections dominate because of their small vertical apertures. We find the threshold current is $I=1.8 \mathrm{~A}$, comfortably above the nominal current.

\section{Multibunch transverse instability}

The resistive wall impedance is often the dominant contributor to the transverse coupled bunch instability in storage rings. Assuming only this source of impedance, the growth rate of the instability can be estimated as [47]

$$
\Gamma=\frac{c\left(I / I_{A}\right)}{4 \gamma \sqrt{\mathcal{C}\left(1-\left[\nu_{y}\right]\right)}}\langle\beta A\rangle,
$$

where

$$
\langle\beta A\rangle=\frac{4}{\sqrt{\pi Z_{0}}} \sum_{i} \frac{\ell_{i} \beta_{y, i}}{b_{i}^{3} \sqrt{\sigma_{c, i}}}
$$

$\left[\nu_{y}\right]$ being the fractional part of the vertical tune. Here the beam pipe is again assumed to be round with radius $b$.

For the growth rate calculation we again use the information in Table VI, letting the vertical half aperture be $b$. Again the undulator and wiggler sections dominate due to the small vertical aperture. We find that the total growth rate $\Gamma=1.4 \mathrm{~ms}^{-1}$, equivalent to a growth time of 99 turns, which should be not too difficult to control with feedback.

\section{CONCLUSION}

In this paper, we have most significantly developed a systematic method based on 4th-order geometric achromats to design an USR where the sextupole magnets are the dominant sources of the nonlinearity. One may choose a different achromat for the design of the lattice; however, our methodology is still applicable. In fact, we know that there are many similar solutions of 4th-order achromats. Since they are not quite relevant to the design of PEP-X, we chose not to present them in this paper.

To make a 4th-order geometric achromat, we chose to eliminate all three tune shift terms in the Lie generator $f_{4}$. In general, this choice may not be suitable for other lattices. Our method can be easily modified to have any values of those three terms. This will allow us to have full control of the size and orientation of the beam footprint in the tune space of the transverse dimensions.

Our design of the PEP-X USR utilizes the existing PEPII tunnel, its high power and low emittance injector, and much of the PEP-II rf system. Perhaps most importantly, the design does not rely on new technology developments and is therefore essentially ready to be built.

Looking forward, there are many interesting topics that can be studied to improve our design. Among them, the most urgent one is to search an efficient and robust method to obtain round beams in electron storage rings.

\section{ACKNOWLEDGMENTS}

We thank K. Kubo for performing SAD IBS calculations and A. Xiao for helping us understand IBS theory with coupling. This work was supported by the Department of Energy under Contract No. DE-AC02-76SF00515.

TABLE VII. The Lie factor $f_{4}$ for two families of sextupoles in the PEP-X achromat.

\begin{tabular}{lcc}
\hline \hline Coefficients $\left[\mathrm{m}^{-1}\right]$ & Analytical & Numerical \\
\hline$\left(a_{x} a_{x}^{+}\right)^{2}$ & $-8.6457 \times 10^{5}$ & $-8.6453 \times 10^{5}$ \\
$\left(a_{y} a_{y}^{+}\right)^{2}$ & $-1.8251 \times 10^{6}$ & $-1.8264 \times 10^{6}$ \\
$\left(a_{x} a_{x}^{+}\right)\left(a_{y} a_{y}^{+}\right)$ & $+2.6396 \times 10^{6}$ & $+2.6405 \times 10^{6}$ \\
$\left(a_{x}\right)^{2}\left(a_{y}^{+}\right)^{2}$ & $(-1.2405-0.00042 i) \times 10^{6}$ & $(-1.2389-0.00036 i) \times 10^{6}$ \\
$\left(a_{x}^{+}\right)^{2}\left(a_{y}\right)^{2}$ & $(-1.2405+0.00042 i) \times 10^{6}$ & $(-1.2389+0.00036 i) \times 10^{6}$ \\
\hline \hline
\end{tabular}




\section{APPENDIX A: MULTIFAMILIES OF SEXTUPOLES IN ARC}

Naturally, we would like to extend the calculation to multiple families of sextupoles in the achromat. While we can use Eq. (3.17) to compute the contribution within any family, the new kind of contribution we need to calculate is the crossing pairs between any two families. For the PEP-X case, we compute 64 brackets and then add them up. The expression is rather complicated but the property of the cancellation is magically retained as shown:

$$
\begin{aligned}
& f_{4}=-\frac{1}{8} S_{1} S_{2} \sqrt{\beta_{x 1} \beta_{x 2}}\left(2 \beta _ { x 1 } \beta _ { x 2 } \left[3(1+\sqrt{2}) \cos \left(\psi_{x 1}-\psi_{x 2}\right)+3 \sin \left(\psi_{x 1}-\psi_{x 2}\right)+(\sqrt{2}-1) \cos 3\left(\psi_{x 1}-\psi_{x 2}\right)\right.\right. \\
& \left.+\sin 3\left(\psi_{x 1}-\psi_{x 2}\right)\right]\left(a_{x} a_{x}^{+}\right)^{2}+2 \beta_{y 1} \beta_{y 2}\left[4(1+\sqrt{2}) \cos \left(\psi_{x 1}-\psi_{x 2}\right)+(\sqrt{2}-1) \cos \left(\psi_{x 1}-\psi_{x 2}+2 \psi_{y 1}-2 \psi_{y 2}\right)\right. \\
& -(\sqrt{2}+1) \cos \left(\psi_{x 1}-\psi_{x 2}-2 \psi_{y 1}+2 \psi_{y 2}\right)+4 \sin \left(\psi_{x 1}-\psi_{x 2}\right)+\sin \left(\psi_{x 1}-\psi_{x 2}+2 \psi_{y 1}-2 \psi_{y 2}\right) \\
& \left.+\sin \left(\psi_{x 1}-\psi_{x 2}-2 \psi_{y 1}+2 \psi_{y 2}\right)\right]\left(a_{y} a_{y}^{+}\right)^{2}-8\left\{\left(\beta_{x 1} \beta_{y 2}+\beta_{y 1} \beta_{x 2}\right)\left[(1+\sqrt{2}) \cos \left(\psi_{x 1}-\psi_{x 2}\right)+\sin \left(\psi_{x 1}-\psi_{x 2}\right)\right]\right. \\
& +8 \beta_{y 1} \beta_{y 2}\left[(\sqrt{2}-1) \cos \left(\psi_{x 1}-\psi_{x 2}+2 \psi_{y 1}-2 \psi_{y 2}\right)+(\sqrt{2}+1) \cos \left(\psi_{x 1}-\psi_{x 2}-2 \psi_{y 1}+2 \psi_{y 2}\right)\right. \\
& \left.\left.+2 \cos \left(\psi_{x 1}-\psi_{x 2}\right) \sin 2\left(\psi_{y 1}-\psi_{y 2}\right)\right]\right\}\left(a_{x} a_{x}^{+}\right)\left(a_{y} a_{y}^{+}\right)+2\left\{\beta _ { x 1 } \beta _ { y 2 } \left[\cos \left(\psi_{x 1}-\psi_{x 2}\right)\right.\right. \\
& \left.+(-1+i \sqrt{2}) \sin \left(\psi_{x 1}-\psi_{x 2}\right)\right] e^{-2 i\left(\psi_{x 1}-\psi_{y 2}\right)}+\beta_{y 1} \beta_{x 2}\left[\cos \left(\psi_{x 1}-\psi_{x 2}\right)-(1+i \sqrt{2}) \sin \left(\psi_{x 1}-\psi_{x 2}\right)\right] e^{2 i\left(\psi_{y 1}-\psi_{x 2}\right)} \\
& \left.+4 \beta_{y 1} \beta_{y 2}\left[(1+\sqrt{2}) \cos \left(\psi_{y 1}-\psi_{y 2}\right)+\sin \left(\psi_{y 1}-\psi_{y 2}\right)\right] e^{-i\left(\psi_{x 1}+\psi_{x 2}-\psi_{y 1}-\psi_{y 2}\right)}\right\}\left(a_{x}\right)^{2}\left(a_{y}^{+}\right)^{2} \\
& +2\left\{\beta_{x 1} \beta_{y 2}\left[\cos \left(\psi_{x 1}-\psi_{x 2}\right)-(1+i \sqrt{2}) \sin \left(\psi_{x 1}-\psi_{x 2}\right)\right] e^{2 i\left(\psi_{x 1}-\psi_{y 2}\right)}\right. \\
& +\beta_{y 1} \beta_{x 2}\left[\cos \left(\psi_{x 1}-\psi_{x 2}\right)-(1-i \sqrt{2}) \sin \left(\psi_{x 1}-\psi_{x 2}\right)\right] e^{-2 i\left(\psi_{y 1}-\psi_{x 2}\right)}+4 \beta_{y 1} \beta_{y 2}\left[(1+\sqrt{2}) \cos \left(\psi_{y 1}-\psi_{y 2}\right)\right. \\
& \left.\left.\left.+\sin \left(\psi_{y 1}-\psi_{y 2}\right)\right] e^{i\left(\psi_{x 1}+\psi_{x 2}-\psi_{y 1}-\psi_{y 2}\right)}\right\}\left(a_{x}^{+}\right)^{2}\left(a_{y}\right)^{2}\right) \text {. }
\end{aligned}
$$

Here we have used the same notation as for the single family and the subscript 1 or 2 indicating the family number. Clearly, this formula along with Eq. (3.17) ensures that there will be no new type of resonance driving terms that would be added to $f_{4}$ when more families of sextupoles are added in the achromat. Moreover, the same conclusion can be made for the thick sextupole families since a family of thick sextupoles can be considered as a set of families of thin sextupoles.

To give a concrete example, we use again the arc of PEP$\mathrm{X}$ and add a second family of eight sextupoles with a total integrated strength $S=100.0 \mathrm{~m}^{-2}$ located where the optical beta functions $\beta_{x}=4.207 \mathrm{~m}$ and $\beta_{y}=3.172 \mathrm{~m}$ and the phase advances $\mu_{x}=5.8622 \mathrm{rad}$ and $\mu_{y}=3.1416 \mathrm{rad}$ relative to the beginning of the cell. All nonvanishing terms for the two families in the achromat are calculated using Eqs. (3.17) and (A1) and tabulated in Table VII. Once again, we have excellent agreement between the two different approaches.

[1] M. Bei et al., Nucl. Instrum. Methods Phys. Res., Sect. A 622, 518 (2010).

[2] G. Hoffstaetter et al., Cornell Technical Report, 2011.

[3] M. Tigner, Nuovo Cimento 37, 1228 (1965).

[4] P. Elleaume and A. Ropert, Nucl. Instrum. Methods Phys. Res., Sect. A 500, 18 (2003).

[5] K. Tsumaki and N. K. Kumagai, Nucl. Instrum. Methods Phys. Res., Sect. A 565, 394 (2006).
[6] R. Hettel et al., in Proceedings of the 2011 Particle Accelerator Conference, NY, USA (IEEE, New York, 2011), pp. 2336-2338.

[7] M. Borland, in Proceedings of the SRI09, Melbourne, 2009, AIP Conf. Proc. No. 1234 (AIP, New York, 2010), p. 911.

[8] Y. Jing, S. Y. Lee, and P.E. Sokol, in Proceedings of the 2011 Particle Accelerator Conference, NY, USA (Ref. [6]), pp. 781-783.

[9] S.C. Leemann, A. Andersson, M. Eriksson, L.-J. Lindgren, E. Wallén, J. Bengtsson, and A. Streun, Phys. Rev. ST Accel. Beams 12, 120701 (2009).

[10] Y. Nosochkov et al., in Proceedings of IPAC2011, San Sebastian, Spain (EPS-IAG, Geneva, 2011), pp. 3068-3070.

[11] M.-H. Wang et al., in Proceedings of IPAC2011, San Sebastian, Spain (Ref. [10]), pp. 3065-3067.

[12] Y. Cai, Nucl. Instrum. Methods Phys. Res., Sect. A 645, 168 (2011).

[13] K. L. Brown and R.V. Servranckx, Nucl. Instrum. Methods Phys. Res., Sect. A 258, 480 (1987).

[14] A. Streun, OPA lattice design code [http://people.web.psi .ch/streun/opa/].

[15] M. Borland et al., Multi-objective direct optimization of dynamic acceptance and lifetime for potential upgrades of the advanced photon light source. APS LS 319, Argonne, 2010.

[16] K.-J. Kim, in Characteristics of Synchrotron Radiation, AIP Proc. No. 184 (AIP, New York, 1989), pp. 565632.

[17] K. Balewski et al., DESY Report No. DESY 2004-035, 2004. 
[18] J. Ablett et al., NSLS-II conceptual design report, Technical report, Brookhaven, 2006 [http://www.bnl.gov/ nsls2/project/CDR].

[19] K. Bane et al., SLAC Report No. SLAC-PUB 13999 , 2010.

[20] M. Sands, SLAC Report No. SLAC 121, 1970.

[21] L. C. Teng, Fermilab Report No. TM-1269, 1984.

[22] M. Sommer, LAL Report No. LAL/RT/83-15, 1983.

[23] A. J. Dragt, J. Opt. Soc. Am. 72, 372 (1982).

[24] J. Irwin, Nucl. Instrum. Methods Phys. Res., Sect. A 298, 460 (1990).

[25] R.D. Ruth, in Single Particle Dynamics in Circular Accelerator, AIP Proc. No. 153 (AIP, New York, 1987), pp. 150-235.

[26] M. Berz, Part. Accel. 24, 109 (1989).

[27] A. J. Dragt and J. M. Finn, J. Math. Phys. (N.Y.) 17, 2215 (1976).

[28] M. Berz, E. Forest, and J. Irwin, Part. Accel. 24, 91 (1989).

[29] Alex J. Dragt, Nucl. Instrum. Methods Phys. Res., Sect. A 258, 339 (1987).

[30] Handbook of Accelerator Physics and Engineering, edited by A. Chao and M. Tigner (World Scientific, Singapore, 2006), 3rd printing.

[31] K. Halbach, J. Phys. (Paris) 44, C1-211 (1983).

[32] Y. Cai, M. Donald, J. Irwin, and Y. T. Yan, SLAC Report No. SLAC-PUB 7642 (1997).

[33] M. Borland, APS Technical Report No. LS-287, 2000.

[34] Y. Cai, SLAC Report No. SLAC-PUB 11084, 2005.
[35] PEP-II: An asymmetric B factory, SLAC Report No. 418, 1993.

[36] SAD optics program written by K. Oide.

[37] J.D. Bjorken and S. K. Mtingwa, Part. Accel. 13, 115 (1983).

[38] S. Nagaitsev, Phys. Rev. ST Accel. Beams 8, 064403 (2005).

[39] K. Bane, in Proceedings of the 8th European Particle Accelerator Conference, Paris, 2002 (EPS-IGA and CERN, Geneva, 2002), pp. 1443-1445.

[40] T. Raubenheimer, Part. Accel. 45, 111 (1994); Report No. SLAC-PUB-5790.

[41] K. Kubo and K. Oide, Phys. Rev. ST Accel. Beams 4, 124401 (2001).

[42] H. Brück, Accelérateurs Circulaires de Particules; introduction à la théorie, Saclay, 1966.

[43] A. Piwinski, in Handbook of Accelerator Physics and Engineering, edited by A. Chao and M. Tigner (World Scientific, Singapore, 2006), pp. 142-144, 3rd printing.

[44] A. Piwinski, DESY Report No. 98-179, 1998.

[45] K. Bane, Y. Cai, and G. Stupakov, Phys. Rev. ST Accel. Beams 13, 104402 (2010).

[46] S. Krinsky, Brookhaven Report No. 75019-2005-IR, 2005.

[47] A. Wolski et al., LBNL Report No. 59449, 2006.

[48] T. Tanaka and H. Kitamura, J. Synchrotron Radiat. 8, 1221 (2001). 